\title{
Observations of the Polarisation of the Anomalous Microwave Emission: A Review
}

\author{
J. A. Rubiño-Martín, ${ }^{1,2}$ C. H. López-Caraballo, ${ }^{1,2}$ R. Génova-Santos, ${ }^{1,2}$ and R. Rebolo ${ }^{1,2}$ \\ ${ }^{1}$ Instituto de Astrofísica de Canarias (IAC), C/Vía Láctea s/n, 38200 La Laguna, Tenerife, Spain \\ ${ }^{2}$ Departamento de Astrofísica, Universidad de La Laguna, 38206 La Laguna, Tenerife, Spain
}

Correspondence should be addressed to J. A. Rubiño-Martín, jose.alberto.rubino@iac.es

Received 13 August 2012; Accepted 10 October 2012

Academic Editor: Clive Dickinson

Copyright (C) 2012 J. A. Rubiño-Martín et al. This is an open access article distributed under the Creative Commons Attribution License, which permits unrestricted use, distribution, and reproduction in any medium, provided the original work is properly cited.

The observational status of the polarisation of the anomalous microwave emission (AME) is reviewed, both for individual compact Galactic regions as well as for the large-scale Galactic emission. There are six Galactic regions with existing polarisation constraints in the relevant range of 10-40 GHz: four dust clouds (Perseus, $\rho$ Ophiuchi, LDN1622, and Pleiades) and two HII regions (LPH96 and the Helix nebula). These constraints are discussed in detail and are complemented by deriving upper limits on the polarisation of the AME for those objects without published WMAP constraints. For the case of large-scale emission, two recent works, based on WMAP data, are reviewed. Currently, the best constraints on the fractional polarisation of the AME, at frequencies near the peak of the emission (i.e., $20-30 \mathrm{GHz})$, are at the level of $\sim 1 \%(95.4 \%$ confidence level). Finally, we compare these constraints with the predictions of some theoretical AME models and discuss the possible impact of polarised AME on future primordial B-mode experiments.

\section{Introduction}

The anomalous microwave emission (hereafter AME) is an excess of dust-correlated microwave $(10-60 \mathrm{GHz})$ emission $[1,2]$ which cannot be explained in terms of the standard physical mechanisms of continuum emission in this frequency range: synchrotron, free-free, and/or thermal dust emission. This AME has been measured by multiple cosmic microwave background $(\mathrm{CMB})$ experiments on both large scales (e.g., COBE [1]; OVRO [2]; Saskatoon [3]; 19 GHz [4]; Python V [5]; Tenerife [6, 7]; GBT [8-10]; COSMOSOMAS [11]; WMAP [12-17]; Planck [18, 19]) as well as in pointed observations of specific Galactic dust clouds (e.g., COSMOSOMAS [20, 21]; CBI [22-24]; AMI [25-27]; VSA $[28,29]$; RATAN-600 [30]; 8 GHz [31, Conklin data]; Planck [18]). Moreover, recent studies also show evidence of AME in nearby galaxies [32, 33]. A comprehensive review of the status of the AME measurements in intensity can be found in this special issue.

Among the various physical mechanisms proposed to explain the AME, electric-dipole emission [34] from very small (less than $10^{3}$ atoms) rapidly rotating $\left(\sim 1.5 \times 10^{10} \mathrm{~s}^{-1}\right)$ dust grains in the interstellar medium (the so-called "spinning dust") appears to be the most favoured mechanism $[18,20,22,35,36]$. However, there are other possible explanations in the literature. For example, magnetic dipole emission [37] from vibrating "magnetic dust" grains has been also discussed. A detailed theoretical description of these models can be found elsewhere [34, 37-42]. A review on the subject is also included in this special issue.

Measurements of the polarisation of the AME may constitute a key tool to distinguish between these models. The polarisation degree of spinning dust emission is predicted to be very small [38], reaching a maximum (6-7\%) around $2-3 \mathrm{GHz}$, and dropping rapidly towards high frequencies (e.g., about $3 \%$ polarisation at $10 \mathrm{GHz}$ and less than $0.5 \%$ above $30 \mathrm{GHz}$ ). Magnetic dust is expected to be, in general, polarised at much higher levels, and more importantly, the predicted frequency behaviour is different [37]. Recently, detailed predictions for the expected polarisation of the magnetic dipole emission, both for aligned free-flying magnetic nanoparticles and for nonspherical silicate host with 
randomly oriented magnetic inclusions, have been reported [42]. They showed that the level of linear polarisation depends strongly on the degree of alignment between the dust grains and the magnetic field, having zero polarisation for random alignments. Furthermore, in cases with magnetic inclusions, the polarisation level can be greater than $5 \%$ at $30 \mathrm{GHz}$, while in the range $10-20 \mathrm{GHz}$ all models have polarisation degrees lower than 1\% (depending on the magnetic inclusions). A relevant prediction is that the polarisation direction can be reversed in this second case with magnetic inclusions, which could be used as an observational tracer to distinguish between these two models.

In this paper, we present an overview of the current observational status of the measurements of the polarisation of the AME. Although there is little information in the literature on this subject, mainly because the polarisation degree of the AME is known to be very small and thus difficult to measure, it is a field with a rapidly increasing activity. In addition to the importance of understanding the physical processes responsible of the emission in the interstellar medium, the characterisation of all polarised astrophysical foregrounds in the microwave range is very relevant for future $\mathrm{CMB}$ experiments aiming to detect a primordial B-mode signal $[43,44]$.

The structure of the paper is as follows. Section 2 presents some practical issues related to polarisation measurements. The current status of the AME polarisation constraints is presented in the following two sections. In Section 3, we review the six Galactic regions where measurements (or upper limits) have been set in the $10-60 \mathrm{GHz}$ frequency range: Perseus, LPH96, Helix Nebulae, $\rho$ Ophiuchi, LDN1622, and Pleiades. In Section 4, we discuss the current constraints on the polarisation of the diffuse (large-scale) Galactic AME emission. For completeness, we derive in Section 5.2 new constraints on the polarisation of the AME for two of the aforementioned regions (LDN1622 and LPH96) for which WMAP constraints were not available. Finally, the conclusions and implications for future B-mode experiments are presented in Section 6.

\section{Polarisation Measurements}

Here we review some specific methodologies and conventions of the polarisation measurements. First of all, we will be reporting only linear polarisation measurements, as the physical mechanisms considered in this frequency range do not produce circular polarisation $(V=0)$.

Linear polarisation can be described in terms of two Stokes parameters [45], $Q$ and $U$. While the intensity $(I)$ is a scalar quantity, $Q$ and $U$ are not: they depend on the selected reference frame. Unless otherwise stated, the values quoted in this paper are referred to Galactic coordinates, and the HEALPix polarisation convention [46] is used (i.e., for a given point on sky, $y$-axis points towards east, and $x$-axis points south). If, for a certain measurement, $(Q, U)$ are given in another reference system which is rotated by an angle $\theta$ with respect to the previous one, the Stokes parameter in our new frame can be obtained as

$$
\begin{gathered}
Q^{\prime}=\cos (2 \theta) Q+\sin (2 \theta) U, \\
U^{\prime}=-\sin (2 \theta) Q+\cos (2 \theta) U .
\end{gathered}
$$

Flux extraction methods used for AME regions are similar to those used for intensity measurements in $\mathrm{CMB}$ studies, but now directly applied to the $Q$ and $U$ maps. The most widely used method for polarisation constraints in compact regions is the aperture photometry (e.g., [4749]), but in principle, other methods could be applied (for a review, see [50]). For example, fitting methods based on different spatial profiles are often used for extracting the intensity in AME regions: either by fitting the beam profile for point-like objects (e.g., [51-53]), or fitting more complex shapes (ellipsoids, multiple Gaussians, etc.) for extended objects (e.g., [20, 28]). Other techniques, as matched filters [54-56], wavelets [57-59], or the recently proposed filtered fusion technique [60], have been used to detect and to measure the polarisation of point-like objects in CMB maps and could in principle be applied to resolve AME regions.

Throughout this paper, we define the polarised intensity as

$$
P=\sqrt{Q^{2}+U^{2}}
$$

and the fractional polarisation (in per cent units) as

$$
\Pi(\%)=100 \frac{\sqrt{Q^{2}+U^{2}}}{I}=100 \frac{P}{I} .
$$

When placing confidence limits on polarisation measurements, there is an specific issue related to $P$ (and $\Pi$ ) estimates, due to the fact that the posterior distributions of the polarisation level (and angle) do not follow a normal (Gaussian) distribution. This issue is especially relevant for AME measurements, because most of the current constraints are either upper limits or marginal detections with very low $\mathrm{S} / \mathrm{N}$ ratios, and if not properly taken into account, the polarisation fraction estimates could be biased.

The posterior distribution of the polarisation level $P$ was described elsewhere [61, 62]. Here we follow [63], and first, we introduce the so-called Rice (or Rician) distribution for the polarisation, which gives the probability of measuring a polarisation fraction in the range $[P, P+d P]$,

$$
R\left(P \mid P_{0}\right) d P=\frac{P}{\sigma} \exp \left[-\frac{P^{2}+P_{0}^{2}}{2 \sigma^{2}}\right] I_{0}\left(\frac{P P_{0}}{\sigma^{2}}\right) \frac{d P}{\sigma},
$$

where $P_{0}$ stands for the true (underlying) polarisation value of the object that we are observing; $I_{0}$ is the zeroth-order modified Bessel function; $\sigma$ stands for the uncertainty on the $Q$ (or $U$ ) measurements (i.e., $\left\langle Q^{2}\right\rangle=\left\langle U^{2}\right\rangle=\sigma^{2}$ ). Note that this expression was derived assuming that the underlying observables ( $Q$ and $U$ ) follow a Gaussian distribution. The $n$th moment of this Rician distribution is given by

$$
E\left[P^{n}\right]=\left(2 \sigma^{2}\right)^{n / 2} \Gamma\left(1+\frac{n}{2}\right){ }_{1} F_{1}\left(-\frac{n}{2}, 1,-\frac{P_{0}^{2}}{2 \sigma^{2}}\right),
$$


where $\Gamma$ stands for the gamma function, and ${ }_{1} F_{1}$ is the confluent hypergeometric function of the first kind [64]. For example, the mean (or first moment) of the Rician distribution, is thus given by

$$
E[P]=\sigma \sqrt{\frac{\pi}{2}}{ }_{1} F_{1}\left(-\frac{1}{2}, 1,-\frac{P_{0}^{2}}{2 \sigma^{2}}\right) .
$$

Note that this equation has the proper asymptotic limit for large values of $P_{0} / \sigma$, in the sense that $E[P] \rightarrow P_{0}$ for $P_{0}^{2} \gg$ $\sigma^{2}$. However, for low signal-to-noise ratios, the mean of this distribution does not coincide with $P_{0}$.

Once we have the Rician distribution, and for the case of a single polarisation measurement, the posterior distribution is given, according to the Bayes' theorem, by

$$
\begin{aligned}
f\left(P_{0} \mid P\right) & =\frac{R\left(P \mid P_{0}\right)}{\int R\left(P \mid P_{0}\right) d P_{0}} \\
& =\sigma^{-1} \sqrt{\frac{2}{\pi}} \exp \left[-\frac{P_{0}^{2}}{2 \sigma^{2}}\right] \exp \left[-\frac{P^{2}}{4 \sigma^{2}}\right] \frac{I_{0}\left(P P_{0} / \sigma^{2}\right)}{I_{0}\left(P^{2} / 4 \sigma^{2}\right)}
\end{aligned}
$$

for the case of a uniform prior in $P_{0}$. This function is plotted in Figure 1 for several values of the measured polarisation $(P)$. Direct integration of (7) can be used to provide an estimate of the true polarisation, $\hat{P}$, as well as to define the confidence intervals. For example, if we define the cumulant distribution $F(P)$ as

$$
F(P)=\int_{0}^{P} f\left(x \mid P_{\mathrm{obs}}\right) d x
$$

for a certain observed polarisation value $P_{\text {obs }}$, then the 95\% confidence limit $P_{u}$ is derived by solving the implicit equation $F\left(P_{u}\right)=0.95$.

For practical purposes, the maximum likelihood solution for $\hat{P}$ can be approximated, in the two limits of low and high $\mathrm{S} / \mathrm{N}$ ratios, by these simple expressions:

$$
\begin{gathered}
\hat{P}=0, \quad \text { for } \frac{P}{\sigma}<\sqrt{2}, \\
\hat{P} \approx \sqrt{P^{2}-\sigma^{2}}, \quad \text { for } \frac{P}{\sigma} \gtrsim 3 .
\end{gathered}
$$

We note that, in practice, this posterior distribution can be also evaluated numerically by using Monte-Carlo simulations based on the noise properties of the measurements (e.g., $[48,49])$. This Monte-Carlo procedure permits us to include not only more complex noise properties, but also the uncertainty in the intensity measurements when deriving the $\Pi$ estimates. The upper limits reported in Section 5 use this Monte-Carlo approach.

\section{Polarisation of the AME in Galactic Regions}

Out of the list of compact Galactic regions showing evidence for AME, we have identified in the literature only six with published polarisation constraints: four dust clouds and two HII regions. In order to provide the constraints on $P$

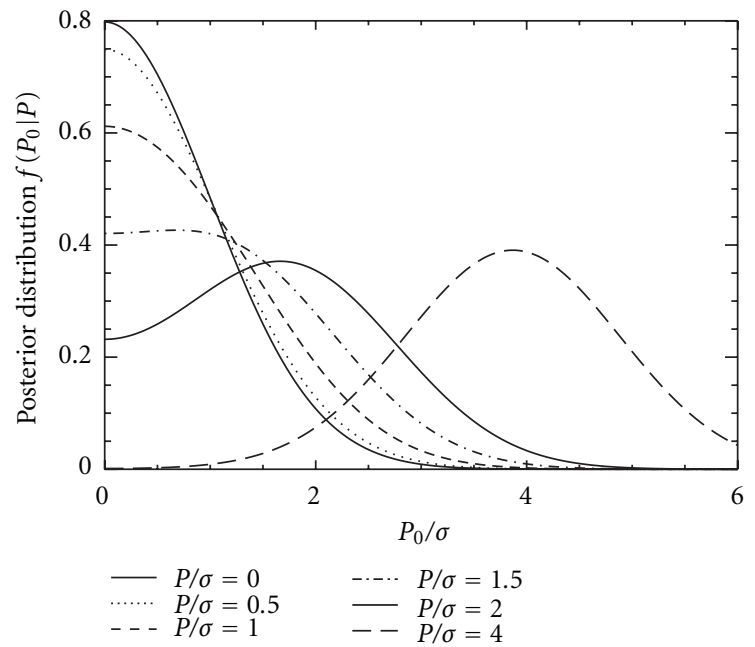

Figure 1: Posterior distribution function from the Rician distribution and for the case of a single polarisation measurement, from (7). Note that for measured polarisation values $P / \sigma<\sqrt{2}$, the posterior distribution (as a function of the true polarisation, $P_{0} / \sigma$ ) peaks at zero polarisation.

and $\Pi$ and for completeness, we also briefly summarise the status of the intensity measurements of those regions. All values described throughout this section are included both in Table 1 and Figure 2.

\subsection{Dust Clouds}

3.1.1. G159.6-18.5 (Perseus). The Perseus molecular complex is a giant molecular cloud located at a distance of $260 \mathrm{pc}$ [68]. The G159.6-18.5 dust feature is our region of interest, an expanding HII bubble that has emerged from the outer edge of the cloud $[69,70]$.

The first detection of AME in G159.6-18.5 was presented in [20] and used the COSMOSOMAS experiment $[11,71]$ $(10-18 \mathrm{GHz})$ and the WMAP $(23-94 \mathrm{GHz})$ data. The spectral energy distribution (hereafter SED) shows a clear emission excess (e.g., $\sim 11.3 \mathrm{Jy}$ and $\sim 27.2 \mathrm{Jy}$ at 11 and $16 \mathrm{GHz}$, resp.), which is well fitted by a spinning dust model in the frequency range $10-60 \mathrm{GHz}$ [34]. A detailed review of the AME in this region is also presented in this special issue.

Using the COSMOSOMAS data at $11 \mathrm{GHz},[65]$ reported a marginal detection of polarised emission in this region, which in principle could be ascribed to AME. Taken as a face value, the reported polarisation fraction $\left(\Pi=3.4_{-1.9}^{+1.5} \%\right.$ at the 95\% confidence level) favours electric dipole emission [34] over dipole magnetic emission [37], as the physical mechanism responsible of the observed polarisation. However, a careful study has to be carried out at lower frequencies to confirm the spinning dust hypothesis. Recently, and using new data from the Effelsberg telescope at $2.7 \mathrm{GHz}(11 \mathrm{~cm})$, [72] proposed that G159.6-18.5 acts as a Faraday Screen (FR) which rotates the polarisation angle of the background emission, and this might contribute significantly to the COSMOSOMAS measurement at $11 \mathrm{GHz}$. 
TABLE 1: Summary of the current constraints on the fractional polarisation ( $\Pi$ ) of the AME both for individual Galactic objects and for large-scale (diffuse) measurements. Columns 1 to 3 indicate the region, the experiment used for this particular constraint, and the angular resolution, respectively. The following four columns indicate the constraints on the fractional polarisation ( $\Pi$ ), separated according to the frequency band for an easier comparison. When quoting upper limits, the $95 \%$ confidence level is used. Last column provides the relevant references.

\begin{tabular}{|c|c|c|c|c|c|c|c|}
\hline Name & Experiment & Resolution & $\begin{array}{c}\prod(9-11 \mathrm{GHz}) \\
{[\%]}\end{array}$ & $\begin{array}{c}\prod(22 \mathrm{GHz}) \\
{[\%]}\end{array}$ & $\begin{array}{c}\text { П }(30-33 \mathrm{GHz}) \\
{[\%]}\end{array}$ & $\begin{array}{c}\prod(40 \mathrm{GHz}) \\
{[\%]}\end{array}$ & References \\
\hline \multicolumn{8}{|c|}{ Galactic AME regions } \\
\hline G159.6-18.5 & COSMOSOMAS & $1^{\circ}$ & $3.4_{-1.9}^{+1.5}$ & & & & {$[65]$} \\
\hline$"$ & WMAP-7 & $1^{\circ}$ & & $<1.01$ & $<1.79$ & $<2.69$ & {$[48]$} \\
\hline$”$ & WMAP-7 & $1^{\circ}$ & & $<1.4$ & $<1.9$ & $<4.7$ & {$[49]$} \\
\hline$\rho$ Ophiuchi & CBI & $\sim 9^{\prime}$ & & & $<3.2$ & & {$[24]$} \\
\hline$”$ & WMAP-7 & $1^{\circ}$ & & $<1.7$ & $<1.6$ & $<2.6$ & {$[49]$} \\
\hline LDN1622 & GBT & $\sim 1.3^{\prime}$ & $<2.7$ & & & & {$[10]$} \\
\hline$”$ & WMAP-7 & $1^{\circ}$ & & $<2.6$ & $<4.8$ & $<8.3$ & This work \\
\hline Pleiades & WMAP-7 & $1^{\circ}$ & & $<12.2$ & $<32.0$ & $<95.8$ & {$[21]$, this work } \\
\hline LPH96 & CBI & $\sim 9^{\prime}$ & & & $<10$ & & {$[23]$} \\
\hline$”$ & WMAP-7 & $1^{\circ}$ & & $<1.3$ & $<2.5$ & $<7.4$ & This work \\
\hline Helix & CBI & $\sim 9^{\prime}$ & & & $<8$ & & {$[66]$} \\
\hline \multicolumn{8}{|c|}{ Diffuse Galactic AME } \\
\hline All sky & WMAP-3 & $1^{\circ}$ & & $<1$ & $<1$ & $<1$ & {$[67]$} \\
\hline All sky & WMAP-5 & $1^{\circ}$ & & $<5$ & & & {$[16]$} \\
\hline
\end{tabular}

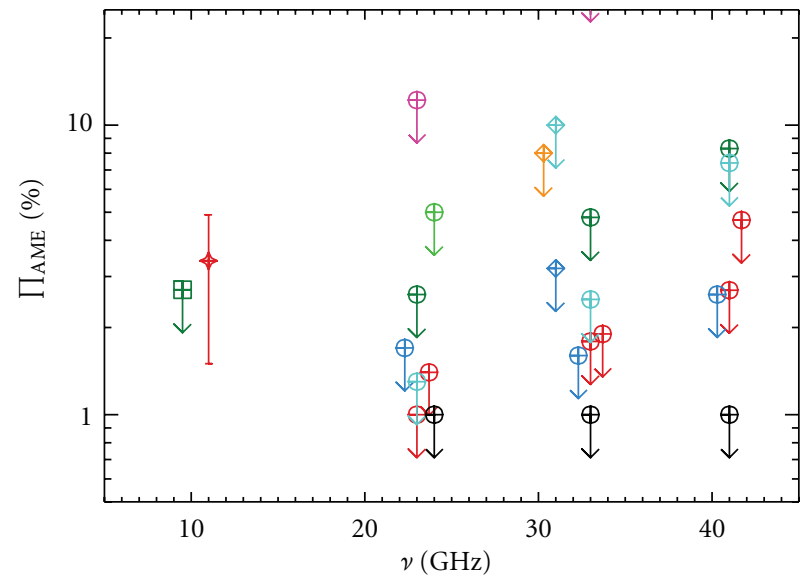

Galactic regions:

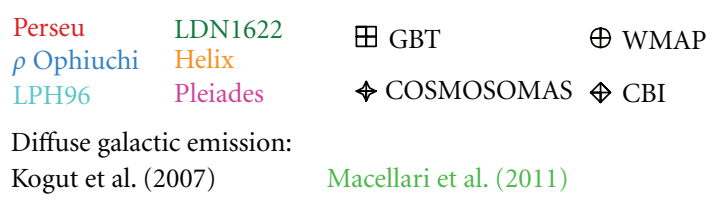

Figure 2: Summary of the current constraints on the fractional polarisation ( $\Pi$ ) of the AME. Data points are taken from Table 1. All upper limits are given at the $95 \%$ confidence level.

Later, [48] studied the polarisation properties of the AME in G159.6-18.5 using the WMAP-7 data, putting constraints on the polarisation fraction of the AME in the region, at an angular resolution of $1^{\circ}$, and using an aperture photometry method on a circular aperture with radius $r_{0}=2^{\circ}$. All measurements in the five WMAP frequency bands were found to be compatible with zero polarisation, and thus, only upper limits for $P$ and $\Pi$ were reported. At $23 \mathrm{GHz}$, they reported $P_{\mathrm{AME}}<0.48 \mathrm{Jy}$ and $\Pi_{\mathrm{AME}}<1.01 \%$, while at $33 \mathrm{GHz}$, the constraints are $0.86 \mathrm{Jy}$ and $1.79 \%$. Note that at these frequencies, the contribution of a possible FR screen in the region is negligible. Again, these results are consistent with the expected polarisation level from electric dipole emission [38].

More recently, [49] revised the polarisation constraints on the Perseus region using WMAP-7 data, using aperture photometry but with a more detailed treatment of the WMAP instrumental noise properties. Their constraints, properly corrected for the Rice bias, are $P_{\mathrm{AME}}<0.24 \mathrm{Jy}$ and $\Pi_{\mathrm{AME}}<1.4 \%$ at $23 \mathrm{GHz}$ and $0.30 \mathrm{Jy}$ and $1.9 \%$ at $33 \mathrm{GHz}$ (95\% confidence level). These values are fully consistent with those obtained in [48].

3.1.2. $\rho$ Ophiuchi. The $\rho$ Ophiuchi molecular cloud (e.g., [73-75]) lies within the Gould Belt region, at a distance of $135 \pm 15 \mathrm{pc}$ [76]. A detailed study of the centimetre-wave continuum radiation in this region was presented in [24], who reported an excess of emission over the expected freefree emission level at $31 \mathrm{GHz}$, spatially associated with the $\rho$ Ophiuchi West bright feature, thus evidencing the presence of AME. Using the Planck data [18], a high-accuracy SED has been derived. The best-fitting model for this SED requires a spinning dust contribution with two components: a highdensity $\left(\sim 2 \times 10^{4} \mathrm{~cm}^{-3}\right)$ molecular gas and a low-density $\left(\sim 200 \mathrm{~cm}^{-3}\right)$ atomic gas.

A polarised intensity map at $31 \mathrm{GHz}$ was obtained in $\rho$ Ophiuchi with the CBI interferometer, with a synthesised 
beam of $9.5 \times 8.3 \mathrm{arcmin}^{2}$ [24]. No obvious polarisation was found in this map, so an upper limit of $\Pi<3.2 \%(2-\sigma)$ was derived for the peak central region and $\Pi<16 \%(2-\sigma)$ for the outer parts. For the integrated flux density, it was set an upper limit of $1 \%$ at $3 \sigma$.

Recently, [49] also provided constraints on the polarisation fraction in this region, based on WMAP-7 data, at $1^{\circ}$ scales. The upper limits, after noise-bias correction, are $P_{\mathrm{AME}}<0.43 \mathrm{Jy}$ and $\Pi_{\mathrm{AME}}<1.7 \%$ at $23 \mathrm{GHz} ; P_{\mathrm{AME}}<0.44 \mathrm{Jy}$ and $\Pi_{\mathrm{AME}}<1.6$ at $33 \mathrm{GHz}$ (95\% confidence level).

3.1.3. LDN1622. Located at a distance of $\sim 200 \mathrm{pc}$ [77], Lynds 1622 (hereafter LDN1622) is a dark cloud that lies in the foreground of the Orion B cloud within the Orion East molecular complex [78]. It extends up to $1 \mathrm{pc}$ according to its far-IR emission. Next to LDN1622, we can find a diffuse HII region named Barnard's Loop.

Using the Green Bank Telescope (GBT), [8] reported the first detection of AME in this region, which was later confirmed by [22], using observations with the CBI at $31 \mathrm{GHz}$, the Parkes-MIT-NRAO survey at $4.85 \mathrm{GHz}$, WMAP data $(23-94 \mathrm{GHz})$, and IRAS. The CBI measurement yields a (loss-corrected) flux density of $2.90 \pm 0.04 \mathrm{Jy}$, which is basically dominated by the AME contribution.

The only upper limit on the polarised AME in LDN1622 that can be found in the literature was reported in [10]. The GBT Spectrometer at $9.65 \mathrm{GHz}$ was used to obtain observations in the four Stokes parameters, yielding $Q=$ $35 \pm 30 \mu \mathrm{K}$ and $U=26 \pm 34 \mu \mathrm{K}$ at an angular resolution of $\sim 6^{\prime}$. These values were translated into upper limits on the polarisation using a maximum likelihood approach similar to the one described in Section 2, resulting $P<88 \mu \mathrm{K}$ and $P<$ $123 \mu \mathrm{K}$ at the $95 \%$ and $99.7 \%$ confidence levels, respectively. The constraint on the fractional polarisation at $9.65 \mathrm{GHz}$ is $\Pi<2.7 \%$ (95\% confidence level).

3.1.4. Pleiades Reflection Nebula. The Pleiades reflection nebula lies within the Taurus complex at a distance of $\sim 120 \mathrm{pc}$ [79]. This is a well-known region, and detailed information about the physical properties of the interstellar medium can be found elsewhere (see e.g., [21], and references therein).

The first evidence for AME in the Pleiades reflection nebula was obtained using the WMAP-7 and COSMOSOMAS data [21]. At $23 \mathrm{GHz}$, the flux density $(2.15 \pm 0.12 \mathrm{Jy})$, integrated within a $1^{\circ}$ radius, mainly corresponds to the AME intensity. The polarised fluxes at this frequency, also derived using aperture photometry, are $Q=0.076 \pm 0.071 \mathrm{Jy}$ and $U=-0.056 \pm 0.091 \mathrm{Jy}$. When combining these values with the intensity measurement and taking into account the noise-bias correction described in Section 2, a $2 \sigma$ upper limit on the polarisation fraction of $\Pi<10.8 \%$ was set at $23 \mathrm{GHz}$.

3.2. HII Regions. At radio/microwave frequencies $(\lesssim 100 \mathrm{GHz})$, HII regions are dominated by free-free (thermal bremsstrahlung) emission from ionised plasma with electron temperatures $T_{e} \approx 8000 \mathrm{~K}$. The spectrum of freefree radiation is well understood $[80,81]$, and it is known to be unpolarised. However, a contribution of AME to the SED of these objects could be in principle expected, since ion collisions with grains are predicted to be one of the largest contributors in maintaining the large rotational velocities required to produce spinning dust emission [34]. We now review the polarisation measurements associated with two HII regions showing evidence of AME: LPH96 and the Helix Nebulae.

3.2.1. LPH96. LPH96 is a diffuse HII region [82], for which observations with the Green Bank 43-m Telescope between 5 and $10 \mathrm{GHz}$ [8] suggested a rising spectrum compatible with AME. However, later $\mathrm{CBI}$ observations at $31 \mathrm{GHz}$ [23] showed no evidence for significant AME. Indeed, the spectral index within the CBI band, and between CBI and Effelsberg data at 1.4 and $2.7 \mathrm{GHz}$, was found to be $\beta=$ $2.06 \pm 0.03$, which is consistent with optically thin free-free emission, thus setting an upper limit of $24 \%(2 \sigma)$ on the intensity of AME at $31 \mathrm{GHz}$. A consistent result, showing no evidence of AME, was obtained using the Very Small Array interferometer [28].

In polarisation, [23] reported CBI observations of Stokes $Q$ and $U$ parameters, with a synthesised beam of $7^{\prime} .9 \times$ $6^{\prime} .5$ (FWHM). The polarised emission was found to be negligible, thus setting a $3 \sigma$ upper limit on the total fractional polarisation of $2 \%$. Depending on the actual intensity of the AME, this limit can be translated into an upper limit on the fractional polarisation of the AME. For instance, [23] argue that if $14 \%$ of the total intensity emission at $31 \mathrm{GHz}$ is indeed anomalous, then the polarisation of this component is $\Pi \lesssim 10 \%$ (at the $2 \sigma$ level).

3.2.2. Helix Nebula and Other PNe. The Helix nebula (NGC7293) is an evolved Planetary Nebula (PN), lying at a distance of $\sim 200 \mathrm{pc}$ [83]. Its angular size is about $10^{\prime}$, and its emission extends up to $1 \mathrm{pc}$ [84].

A detailed multifrequency analysis on the region was carried out in [85]. The derived SED shows a dustcorrelated $31 \mathrm{GHz}$ excess over free-free emission, which cannot be explained in terms of a synchrotron component, nor with optically thick knots, nor in terms of ultracold grains. However, one of the most interesting aspects of this detection is that very small grains, thought not to survive in evolved Planetary Nebulae ( $\mathrm{PNe}$ ), have not been detected in NGC7293. Therefore, the presence of AME cannot be explained in terms of electric dipole emission from spinning dust grains, and thus, for this particular case, the magnetic dipole emission from ferromagnetic grains is favoured [85]. Because of this particularity, [66] extended the previous work in the Helix, by considering a more extended sample of 37 PNe. Using CBI measurements, they showed that the $31 \mathrm{GHz}$ flux densities in their PNe sample are systematically higher than the level of optically thin free-free continuum extrapolated from $250 \mathrm{GHz}$. The $31 \mathrm{GHz}$ excess is observed in all $18 \mathrm{PNe}$ with reliable 31 and $250 \mathrm{GHz}$ data and is significant in nine $\mathrm{PNe}$.

Polarisation measurements at $31 \mathrm{GHz}$ were obtained in four objects of the full sample: Helix, NGC7009, NGC1360, and NGC246. No significant polarisation was detected in any of these four PNe, being all Stokes $Q$ and $U$ maps fully consistent with noise. The $99 \%$ upper limits on the total 
polarisation fraction, after correcting for the noise bias as described in Section 2, are 8.5\%, 8.5\%, 17\%, and 2.4\% for NGC7009, NGC1360, NGC246, and NGC7293, respectively.

For the case of the Helix, and since free-free radiation is not polarised, that limit on the total polarisation can be converted into an upper limit for the AME polarisation fraction. For $36-80 \%$ free-free emission at $31 \mathrm{GHz}$, this translates into $\Pi<3.8-12 \%$ for the AME, also at the $99 \%$ confidence level. The more conservative number $(8 \%$ at 2 sigmas) is also included in Table 1.

\section{Polarisation of the Diffuse Galactic AME}

Due to the low-polarisation degree of the AME measured in Galactic regions, most of the component separation methods attempting to separate the AME in polarisation from CMB maps normally assume that this component is unpolarised. Because of this reason, there are only a few constraints in the literature on the polarisation of the (large-scale) diffuse Galactic AME emission. Here we review two papers, both based on WMAP data.

First, [67] used the WMAP 3-year data to constrain the fractional polarisation of the AME. By assuming that the spatial distribution and the polarisation angles of the AME can be traced by dust templates and adopting a spinning dust model [34] for the frequency dependence of the emission, they fitted the polarisation data searching for an AME contribution. Their conclusion is that the spinning dust contribution is less than $1 \%$ of the observed polarisation signal variance in any WMAP band (23-94 GHz).

More recently, [16] used a different approach, based on a cross-correlation analysis between the WMAP 5year polarisation maps and several intensity template maps tracing the Galactic synchrotron, dust, and free-free emissions. They derived the polarisation fraction for all those three components in 48 sky regions (corresponding to the HEALPix pixelisation with $N_{\text {side }}=2$ ). In intensity, the dust-correlated emission is found to be the dominant signal over the entire sky in the 23-94 GHz range, being the anomalous emission clearly detected at 23 and $33 \mathrm{GHz}$. At these two frequencies, the AME is found to be the dominant foreground at low Galactic latitudes $\left(-40^{\circ}<b<10^{\circ}\right)$. The average fractional polarisation of dust-correlated AME at $23 \mathrm{GHz}$ is $3.2 \pm 0.9$ (stat) \pm 1.5 (sys) per cent, or equivalently, $\Pi<5 \%$ at the $95 \%$ confidence level. These two results have been included in Table 1 and Figure 2.

\section{New Constraints}

In the previous sections we have presented a compilation of the up-to-date polarisation measurements of AME in individual regions, as well as in the diffuse Galactic emission, which is summarised in Table 1. Now, we use WMAP 7-year data to expand the spectral range of these constraints in those regions where we have not found WMAP measurements in the literature. For LDN1622, [10] derived an upper limit at $9.65 \mathrm{GHz}$ using data from the GBT telescope, whereas the $31 \mathrm{GHz}$ measurement for LPH96 was obtained by [23] using data from the CBI interferometer. In the Pleiades reflection nebula, [21] presented an upper limit using only the $23 \mathrm{GHz}$ WMAP channel. Here, we complement the polarisation measurements for those three regions using the WMAP maps. We note that the Helix nebula is not included in our study, because of the low flux density and small angular extent of this object, making the AME emission of this region undetectable in WMAP maps.

5.1. Data and Methodology. We use the WMAP 7-year data products [86], which are publicly available in the LAMBDA webpage (http://lambda.gsfc.nasa.gov/) in the HEALPix pixelisation scheme. Maps are provided for each of the five WMAP frequency bands $(\mathrm{K}, \mathrm{Ka}, \mathrm{Q}, \mathrm{V}$, and $\mathrm{W})$, centred at $22.8,33.0,40.7,60.8$, and $93.5 \mathrm{GHz}$. The original angular resolutions of these bands are, respectively, $0.85^{\circ}, 0.65^{\circ}$, $0.51^{\circ}, 0.35^{\circ}$, and $0.25^{\circ}$ although, for consistency, we will work with maps degraded to a common resolution of $1^{\circ}$.

Figures 3, 4, and 5 show maps of the $I, Q$, and $U$ Stokes parameters at LDN1622, LPH96, and the Pleiades reflection nebula, respectively. No clear polarised emission is visible in any of these maps. Thus, we use an aperture photometry integration to derive upper limits on the polarisation fractions. As explained in [48], this is an efficient technique for computing fluxes, or upper limits, in regions of low signal to noise, which at the same time allows a reliable subtraction of the underlying background signal. It consists of an integration of the temperatures of all pixels within a given aperture, after subtracting the mean background level which is calculated by averaging the temperatures of all pixels enclosed in an external annulus. The central coordinates, together with the radii of the inner aperture and of the background ring used in each case, are shown in Table 2. The flux estimate is given by

$$
S_{v}=a(v)\left[\frac{\sum_{i=1}^{n_{1}} T_{i}}{n_{1}}-\frac{\sum_{j=1}^{n_{2}} T_{j}}{n_{2}}\right],
$$

where $n_{1}$ and $n_{2}$ are, respectively, the number of pixels in the circular aperture and in the background annulus, and $T_{i}$ and $T_{j}$ represent the pixel thermodynamic temperatures in the aperture and in the external annulus (here by "temperature" we refer either to total intensity, or temperature in Stokes $Q$ and $U$, when we calculate polarised fluxes). The function $a(\nu)$ gives the conversion factor from temperature to flux,

$$
a(v)=\frac{h^{2} v^{4}}{2 k_{B} T_{0}^{2} c^{2}} \sinh ^{-2}\left(\frac{h \nu}{2 k_{B} T_{0}}\right) n_{1} \Omega_{\mathrm{pix}}
$$

where $h$ and $k_{B}$ are the Planck and Boltzmann constants; $T_{0}=2.725 \mathrm{~K}$ is the CMB temperature today [87]; $\Omega_{\text {pix }}$ stands for the solid angle subtended by a HEALPix pixel (all of them have the same area). 

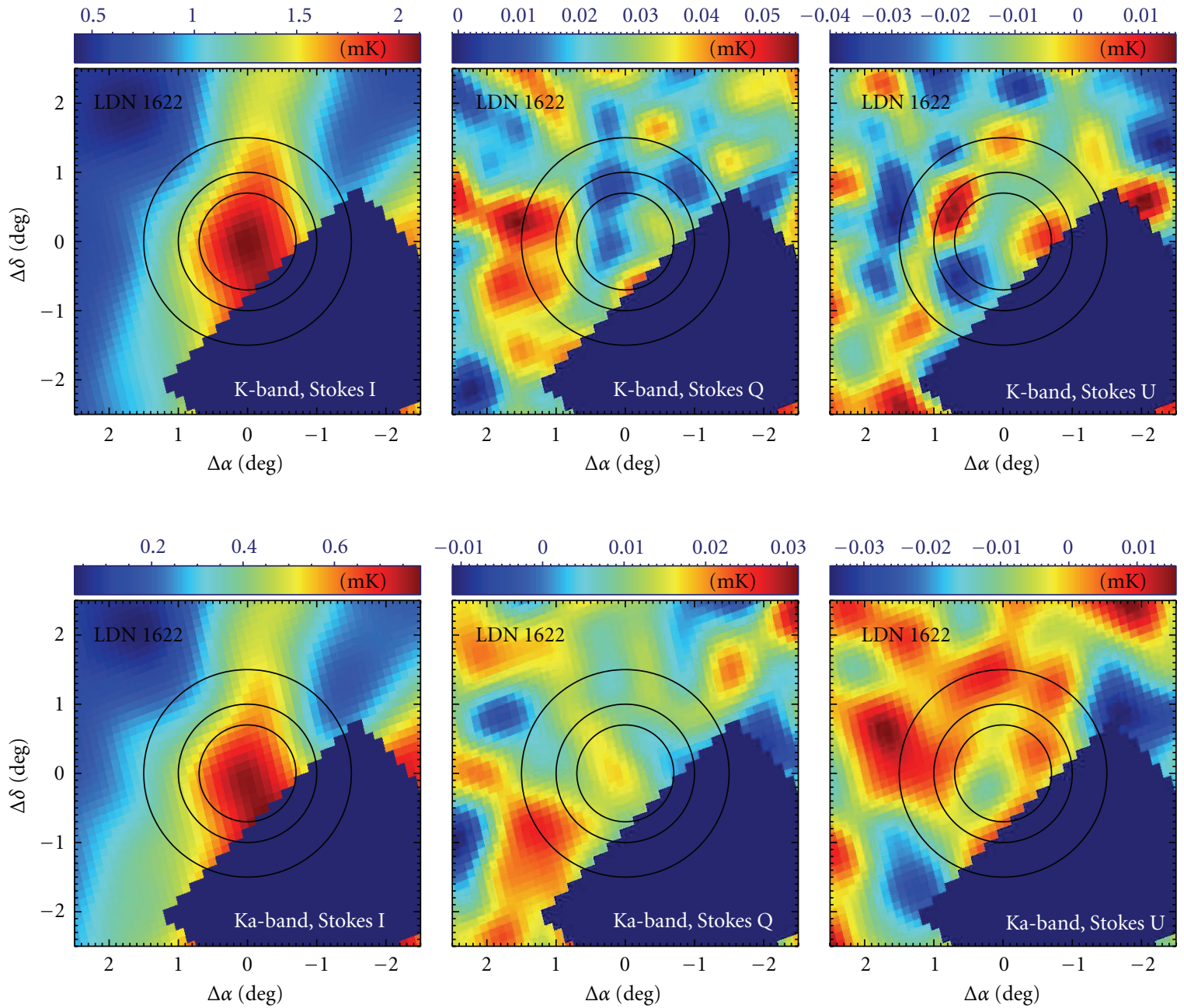

FIGURE 3: The LDN1622 region, as seen by WMAP in Stokes parameters $I$ (left), $Q$ (centre), and $U$ (right column), at $23 \mathrm{GHz}$ (top row) and $33 \mathrm{GHz}$ (bottom row). All maps are displayed at the common angular resolution of $1.0^{\circ}$. The circles $\left(r_{0}=0.7^{\circ}, r_{1}=1.0^{\circ}\right.$, and $\left.r_{2}=1.5^{\circ}\right)$ indicate the main aperture $\left(r_{0}\right)$ and the region for the background correction $\left(r_{1}<r<r_{2}\right)$ used for the aperture photometry method. The masked regions are not included in the flux density determination (see text for details).

The associated error bar for the flux estimator given (10) can be computed analytically, using the pixel-to-pixel covariance, as

$$
\begin{gathered}
\sigma\left(S_{\nu}\right)=a(v)\left[\frac{1}{n_{1}^{2}} \sum_{i, i^{\prime}}^{n_{1}, n_{1}} C\left(\theta_{i i^{\prime}}\right)+\frac{1}{n_{2}^{2}} \sum_{j, j^{\prime}}^{n_{2}, n_{2}} C\left(\theta_{j j^{\prime}}\right)\right. \\
\left.-\frac{2}{n_{1}, n_{2}} \sum_{i, j}^{n_{1} n_{2}} C\left(\theta_{i j}\right)\right]^{1 / 2},
\end{gathered}
$$

where the indices $i$ and $i^{\prime}$ run over pixels in the aperture region, while $j$ and $j^{\prime}$ run over pixels in the background region. In (12), $C\left(\theta_{i j}\right)$ is the pixel-to-pixel 2-point correlation function, which is evaluated for any pair of pixels separated by an angle $\theta_{i j}$, and in general, it includes the $\mathrm{CMB}$, the background emission, and the instrumental noise contributions.

In the case of the total intensity estimates, the two main contributions to the error bar are the instrumental noise and
TABLE 2: Central positions and radii used in the aperture photometry analysis for each region.

\begin{tabular}{lccccc}
\hline Name & $\begin{array}{c}\text { R.A. (J2000) } \\
(\mathrm{deg})\end{array}$ & $\begin{array}{c}\text { Dec. } \\
(\mathrm{deg})\end{array}$ & $\begin{array}{c}r_{0} \\
(\mathrm{deg})\end{array}$ & $\begin{array}{c}r_{1} \\
(\mathrm{deg})\end{array}$ & $\begin{array}{c}r_{2} \\
(\mathrm{deg})\end{array}$ \\
\hline LDN1622 & 88.64 & 2.10 & 0.7 & 1.0 & 1.5 \\
LPH96 & 99.28 & 10.70 & 0.7 & 1.0 & 1.5 \\
Pleiades & 56.24 & 23.78 & 1.0 & 1.7 & 2.0 \\
\hline
\end{tabular}

the background fluctuations, which chiefly arise from the large-scale Galactic emission and from the CMB. In order to account for the two terms, following [49], we estimate the associated flux error bar through the quadratic sum of the standard deviations in the aperture and in the background:

$$
\sigma\left(S_{\nu}^{I}\right) \approx a(v) \sigma\left(T_{\mathrm{bg}}\right)\left[\frac{1}{n_{\mathrm{ap}}}+\frac{1}{n_{\mathrm{bg}}}\right]^{1 / 2},
$$



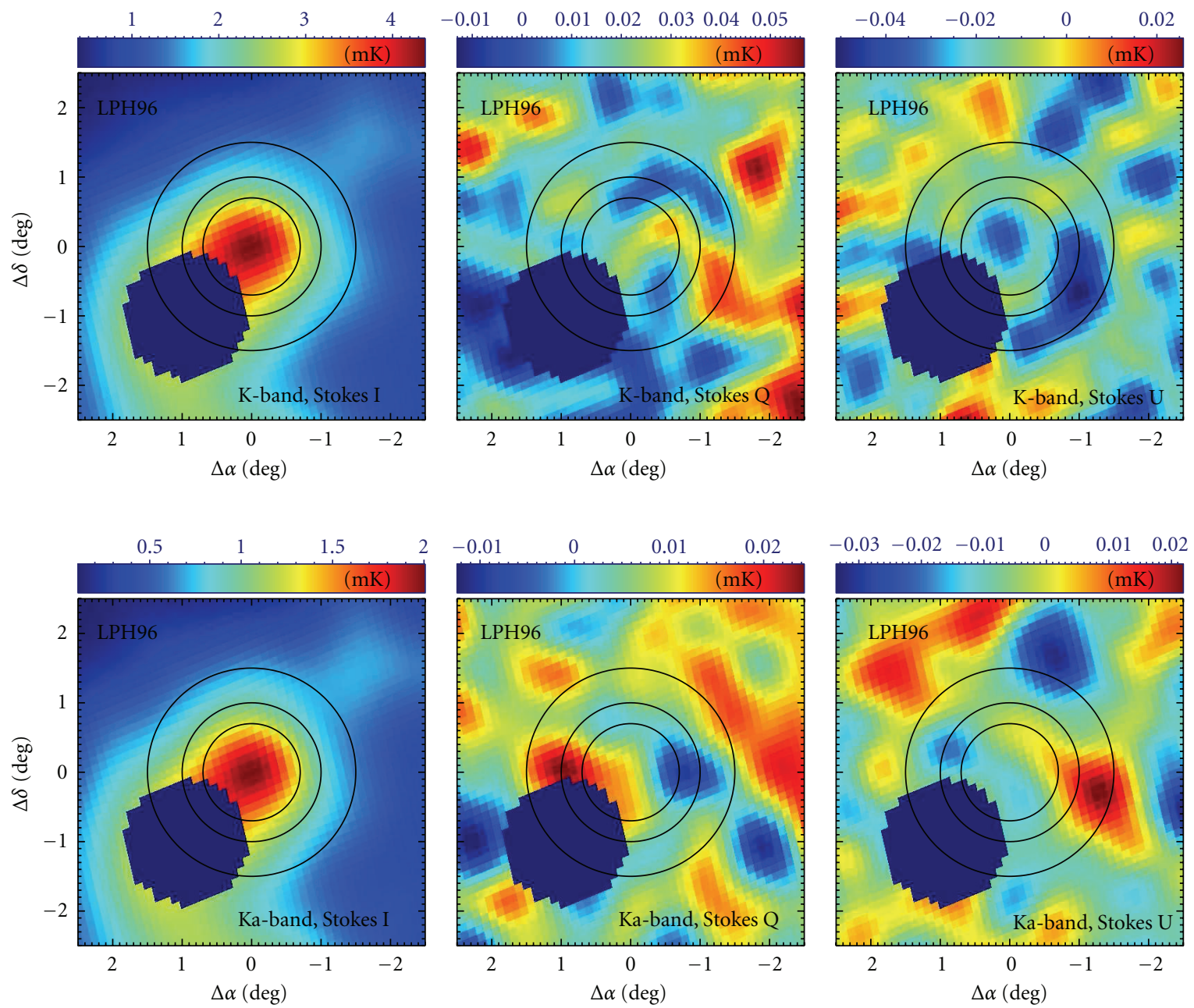

FIgURE 4: The same as Figure 3, but for the LPH96 region.

where $\sigma\left(T_{\mathrm{bg}}\right)$ represents the pixel-to-pixel standard deviation in the background; $n_{\mathrm{ap}}$ and $n_{\mathrm{bg}}$ are the number of independent pixels in the aperture and the background, respectively. Those are calculated as the equivalent number of beams that can be accommodated within each aperture. Note that in (13), we do not include the standard deviation on the aperture, as this will be contaminated by the source contribution.

Conversely, when extracting the $Q$ and $U$ fluxes on WMAP data, we have checked that the contributions of the background fluctuations and the CMB are not so critical. Therefore, in this case, we follow [48] and we use (12), being the correlation function entirely due to instrumental noise. Note that, even in this case of almost uncorrelated noise, we still have a contribution which arises from the smoothing process applied to bring all the maps to a common $1^{\circ}$ angular resolution.

In order to derive polarisation upper limits associated with the AME, we need to have an estimate of the AME total intensity flux at each of the WMAP frequencies, which might be viewed as the residual flux after subtracting the other contributions, namely, the $\mathrm{CMB}$, the free-free, and the thermal dust emissions. We characterise the free-free emission as $I_{\mathrm{ff}}=A_{\nu_{0}}^{\mathrm{ff}}\left(\nu / \nu_{0}\right)^{\beta_{\mathrm{ff}}}$, with $\beta_{\mathrm{ff}}=-0.1$, from low-frequency radio surveys at $0.408 \mathrm{GHz}$ [88], $0.820 \mathrm{GHz}$ [89], and $1.4 \mathrm{GHz}$ [90]. The thermal dust emission is modelled using a modified black-body spectrum, $I_{d}\left(\nu, T_{d}\right)=$ $\tau_{100}(v /(2998 \mathrm{GHz}))^{\beta_{d}} B_{v}\left(T_{d}\right)$, using far-infrared data from the DIRBE satellite between 1249 and $2998 \mathrm{GHz}$ [91]. Finally, a CMB component is fitted to the data using a blackbody curve. The total AME intensity flux is then calculated as $I_{\mathrm{AME}}=I_{\text {total }}-I_{\mathrm{ff}}-I_{d}-I_{\mathrm{cmb}}$, and the final AME polarisation fraction as $\Pi_{\mathrm{AME}}=100 P_{0} / I_{\mathrm{AME}}$, where $P_{0}$ is the debiased polarised flux, which is calculated from the measured polarised flux, $P$, following the formalism presented in Section 2. The uncertainty of those quantities $\left(I_{\mathrm{AME}}\right.$ and $\left.\Pi_{\mathrm{AME}}\right)$ is derived by propagating the uncertainty of the SED fit.

\subsection{Results}

5.2.1. LDN1622. After applying a mask to remove the contamination introduced by the neighbouring Barnard's loop HII region (The region masked for our analysis has a rectangular shape, defined by the following four vertices: v1 : $\left(\right.$ R.A. $=87 . .^{\circ} 07$; Dec. $\left.=2^{\circ} .9\right)$, v2: $\left(\right.$ R.A. $=85^{\circ} .04$; Dec. 

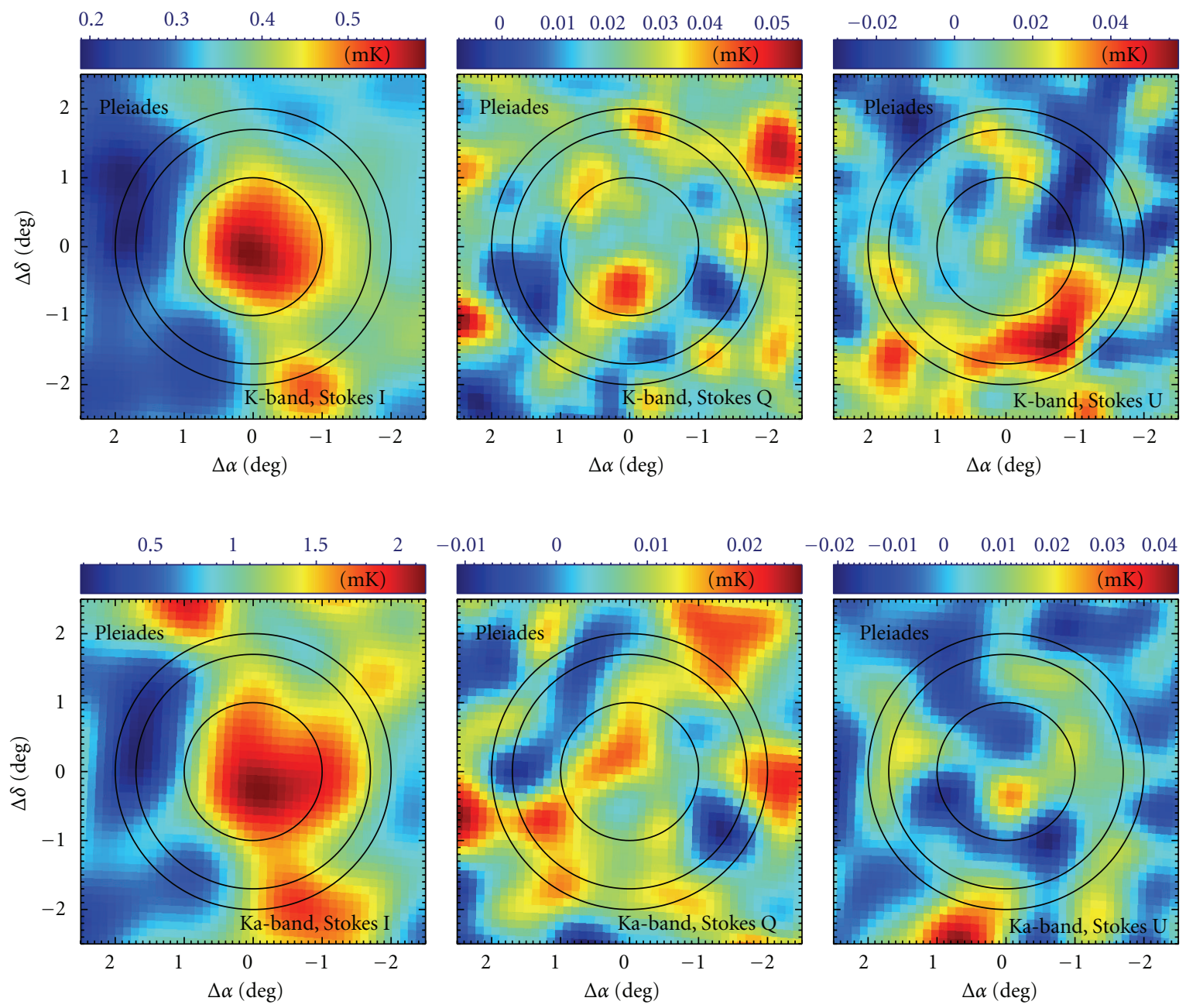

FIgure 5: The same as Figure 3, but for the Pleiades region. The apertures now correspond to the radii $r_{0}=1^{\circ}, r_{1}=1.5^{\circ}$, and $r_{2}=2^{\circ}$.

$\left.=0^{\circ} .79\right), \mathrm{v} 3:\left(\right.$ R.A. $=87^{\circ} .79 ;$ Dec. $\left.:-1^{\circ} .53\right)$, and v4:(R.A. $=89^{\circ} .91$; Dec. $\left.=0^{\circ} .03\right)$.), we get the intensity fluxes listed in Table 3. Our derived fluxes at low frequencies $(1.9 \pm 4.2$ and $1.19 \pm 0.85 \mathrm{Jy}$, at 0.40 and $1.42 \mathrm{GHz}$, resp.), should be considered as upper limits, as they are within the error bars and the maps do not present clear emission. To determine the amplitude of the free-free emission, we instead use the flux density of $0.086 \pm 0.042 \mathrm{Jy}$ obtained by [22] at $5 \mathrm{GHz}$. We then perform a joint fit of the $\mathrm{CMB}$ and thermal dust components using the $94 \mathrm{GHz}$ WMAP flux and the DIRBE fluxes in the three bands, after subtracting the free-free emission corresponding to each band. We fix the emissivity index at the value given by [22], $\beta_{d}=1.7$, and fit for the remaining three parameters, obtaining $T_{d}=17.17 \pm 0.31 \mathrm{~K}$, $\tau_{100}=(9.53 \pm 1.02) \times 10^{-4}$, and $T_{\mathrm{cmb}}=35.60 \pm 6.4 \mu \mathrm{K}$. The final AME fluxes are listed in Table 3.

The $Q$ and $U$ WMAP maps of Figure 3 do not show significant signal towards this region, and the computed fluxes are accordingly compatible with the error bars. We therefore derive the 2-sigma upper limits on the fractional polarisation shown in Table 4 . These constraints, as well as the $2.7 \%$ limit at $9 \mathrm{GHz}$ given by [10], rule out all the models presented in [37] based on magnetic dipole emission from single-domain dust grains (the magnetic field is aligned either parallel or perpendicular to the largest moment of inertia of the grains), all of which predict higher polarisation fractions. However, a lower polarisation could arise when grains are arranged in different magnetic domains.

5.2.2. LPH96. The WMAP maps at the diffuse HII region LPH96 are shown in Figure 4. A circular mask with radius $0^{\circ} .8$ has been applied to remove the contamination from the point source located at position R.A. $(\mathrm{J} 2000)=100^{\circ} .23$ and Dec. $=9^{\circ} \cdot 70$. Significant emission is visible in the lowfrequency maps $(8.4 \pm 4.4$ and $7.2 \pm 1.7 \mathrm{Jy}$ at 0.408 and $1.42 \mathrm{GHz}$, resp.); so we use those values to constrain the amplitude of the free-free component. We perform the joint $\mathrm{CMB}$ and thermal dust fit in the same way as it was applied to LDN1622. In this case, we fix $\beta_{d}=1.7$ and $T_{d}=18.175 \mathrm{~K}$ [9], and fit for the remaining two parameters, obtaining $T_{\mathrm{cmb}}=85.22 \pm 2.66 \mu \mathrm{K}$ and $\tau_{100}=(8.33 \pm 0.13) \times 10^{-4}$.

The residual AME flux at $33 \mathrm{GHz}$ is $6.91 \pm 2.63 \mathrm{Jy}$, which represents about the $50 \%$ of the total (measured) flux. This value supports the results of [8], who first claimed the detection of AME in this region, but apparently contradicts those of [23], who obtained an AME flux of $14 \%$ of the total 
TABLE 3: Stokes $I, Q$, and $U$ measured in LDN1622, LPH96, and the Pleiades.

\begin{tabular}{lcccc}
\hline$v(\mathrm{GHz})$ & $I(\mathrm{Jy})$ & $I_{\mathrm{AME}}(\mathrm{Jy})$ & $Q(\mathrm{Jy})$ & $U(\mathrm{Jy})$ \\
\hline \multicolumn{5}{c}{ LDN1622 } \\
23 & $5.64 \pm 1.90$ & $5.22 \pm 1.90$ & $-0.05 \pm 0.05$ & $0.01 \pm 0.06$ \\
33 & $4.90 \pm 1.70$ & $4.05 \pm 1.72$ & $-0.01 \pm 0.09$ & $-0.04 \pm 0.09$ \\
41 & $4.44 \pm 1.77$ & $3.11 \pm 1.82$ & $-0.05 \pm 0.11$ & $0.04 \pm 0.12$ \\
61 & $4.84 \pm 2.95$ & $1.42 \pm 3.20$ & $0.16 \pm 0.27$ & $0.06 \pm 0.29$ \\
94 & $9.96 \pm 5.89$ & $-0.61 \pm 7.80$ & $0.25 \pm 0.64$ & $0.05 \pm 0.69$ \\
\hline \multicolumn{5}{c}{ LPH96 } \\
23 & $13.95 \pm 2.44$ & $7.86 \pm 2.80$ & $-0.00 \pm 0.05$ & $0.01 \pm 0.06$ \\
33 & $13.65 \pm 2.26$ & $6.91 \pm 2.63$ & $-0.02 \pm 0.08$ & $-0.04 \pm 0.10$ \\
41 & $13.37 \pm 2.28$ & $5.87 \pm 2.63$ & $0.17 \pm 0.11$ & $-0.10 \pm 0.13$ \\
61 & $14.07 \pm 2.49$ & $3.48 \pm 2.80$ & $-0.49 \pm 0.25$ & $0.20 \pm 0.31$ \\
94 & $19.62 \pm 5.02$ & $0.12 \pm 5.19$ & $-0.08 \pm 0.60$ & $0.17 \pm 0.74$ \\
\hline \multicolumn{5}{c}{ Pleiades } \\
23 & $2.60 \pm 0.90$ & $1.94 \pm 0.90$ & $0.08 \pm 0.07$ & $-0.06 \pm 0.09$ \\
33 & $2.55 \pm 1.15$ & $1.20 \pm 1.15$ & $0.06 \pm 0.13$ & $-0.07 \pm 0.17$ \\
41 & $2.64 \pm 1.26$ & $0.63 \pm 1.28$ & $-0.14 \pm 0.20$ & $-0.14 \pm 0.24$ \\
61 & $4.71 \pm 2.49$ & $0.38 \pm 2.52$ & $-0.06 \pm 0.47$ & $-0.58 \pm 0.57$ \\
94 & $9.11 \pm 5.19$ & $-0.52 \pm 5.26$ & $-1.02 \pm 1.16$ & $-1.26 \pm 1.40$ \\
\hline
\end{tabular}

TABLE 4: Upper limits at the 95\% confidence level, on the fractional linear polarisation of the AME measured in LDN1622, LPH96, and Pleiades at the three lowest WMAP frequency bands.

\begin{tabular}{lcccccc}
\hline \multirow{2}{*}{ Region } & \multicolumn{3}{c}{$P_{0}(\mathrm{Jy})$} & \multicolumn{3}{c}{$\Pi_{\mathrm{AME}}(\%)$} \\
& $23 \mathrm{GHz}$ & $33 \mathrm{GHz}$ & $41 \mathrm{GHz}$ & $23 \mathrm{GHz}$ & $33 \mathrm{GHz}$ & $41 \mathrm{GHz}$ \\
\hline LDN1622 & $<0.12$ & $<0.19$ & $<0.23$ & $<2.6$ & $<4.8$ & $<8.3$ \\
LPH96 & $<0.10$ & $<0.17$ & $<0.34$ & $<1.3$ & $<2.5$ & $<7.4$ \\
Pleiades & $<0.19$ & $<0.28$ & $<0.51$ & $<12.2$ & $<32.0$ & $<95.8$ \\
\hline
\end{tabular}

measured flux at $31 \mathrm{GHz}$ with the $\mathrm{CBI}$ interferometer and claimed not to find evidence of AME towards this source. A possible explanation for this inconsistency is given by the fact that [23] focused their analysis on a compact structure $\left(\sim 6^{\prime}\right)$ in the region, while in the present analysis, we obtained the flux density by integrating over an area of $\sim 0.7^{\circ}$, thus also taking into account a possible extended emission which would be resolved out by the CBI interferometer (see [23] for details).

No signal is evident in the $Q$ and $U$ maps at the position of LPH96, and in fact the measured fluxes are compatible with the instrumental noise. We therefore derive the 2-sigma upper limits on the fractional polarisation shown in Table 4. Our result at $33 \mathrm{GHz}$ is about four times more restrictive than the one calculated by [23] at $31 \mathrm{GHz}$.

5.2.3. Pleiades. The intensity and polarisation WMAP maps in the position of the Pleiades reflection nebula are shown in Figure 5. We have followed the same process as in [21], with the only difference that now we calculate errors in the intensity flux using (13). This barely changes the results of the $\mathrm{CMB}$ and thermal dust fits. The fluxes corresponding to total intensity and to polarisation are shown in Table 3 . The AME residual flux at $23 \mathrm{GHz}$ is at a less significance (2.2-sigma) than that obtained in [21], owing to the different approach for calculating error bars. The upper limits on the polarisation fraction shown in Table 4 are less restrictive than those in the other two sources, due to the lower AME intensity.

\section{Discussion}

The observational study of the polarisation of the AME provides a valuable tool to understand the physical mechanism(s) responsible for the AME and to disentangle among the different models proposed in the literature.

From an observational point of view, exploring the spectral and spatial properties of the polarisation of the AME is a challenge because of the expected low polarisation fraction. Despite this difficulty, in the last few years there has been a sustained observational effort, and nowadays the current upper limits (see Table 1) of the AME polarisation fraction are at the level of $\Pi \approx 1 \%$, both for the extended emission and for individual regions. There is only one detection claimed to date, in the Perseus molecular complex [65], but in order to confirm the physical mechanism responsible for the emission, other detections are needed at different wavelengths.

Nevertheless, even if we only have upper limits, we can still significantly constrain some of the models in the literature, in particular those predicting high fractional polarisation degrees. To illustrate this issue, Figures 6 and 7 compare, at $23 \mathrm{GHz}$ and $33 \mathrm{GHz}$, all the upper limits listed in Table 1, with the predictions of some polarisation models, corresponding to the electric dipole (ED) and magnetic dipole (MD) emissions. For the ED case, we show the polarised Cold Neutral Medium spinning dust model proposed by [34]. Note that, as this model is derived under the assumption of negligible saturation effects, this curve corresponds to the maximal values allowed by the paramagnetic mechanism. Levels of $\lesssim 1 \%$ and $\lesssim 0.5 \%$ are expected at $23 \mathrm{GHz}$ and $33 \mathrm{GHz}$, respectively.

For the polarised MD emission we considered two cases: (1) grains with single magnetic domain; (2) grains with magnetic inclusions. For the first case, [37]'s models from perfectly aligned grains consisting of a single magnetic domain are determined by the dotted and dashed lines, for two different grain shapes with axial ratios $1: 2: 2$ and $1: 1.25: 1.5$, respectively. Note that the frequency behaviour depends strongly on the shape and composition of the grains. Recently, [42] showed that the level of linear polarisation can be much lower when the grains are partially aligned, decreasing to zero polarisation for random alignments. Thus, these curves should again be considered as maximal values for this mechanism. In the perfect alignment case, the models predict polarisation fractions between 10 and $20 \%$ at $23 \mathrm{GHz}$ (see Figures 6 and 7).

In the second case, the randomly oriented magnetic inclusions models [42] predict at $20 \mathrm{GHz}$ levels lower than $5 \%$ (depending on the magnetic inclusions). Meanwhile, at $33 \mathrm{GHz}$ they reach levels between $\sim 5 \%$ and $\sim 10 \%$.

The low levels of polarised AME emission allow us to rule out several models based on MD emission, in particular 


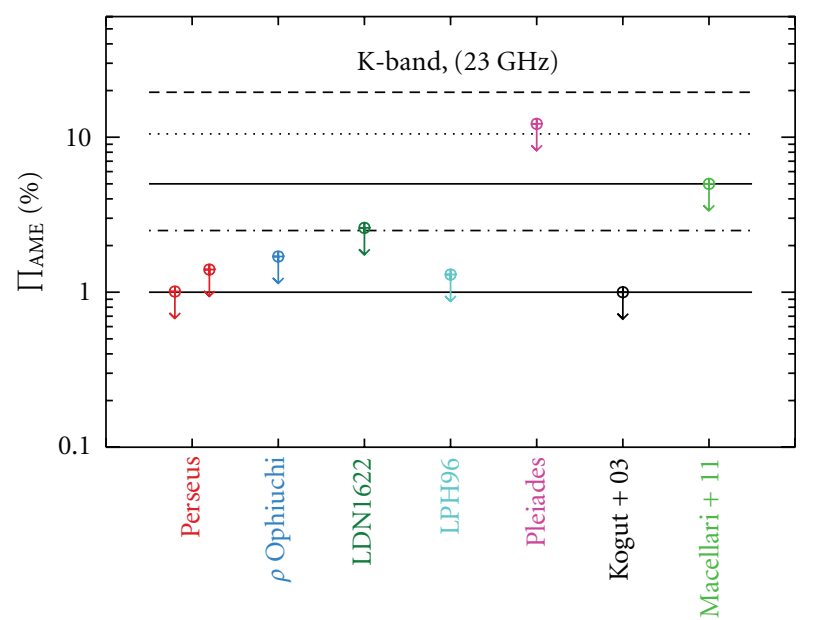

- Electric dipole (ED) -.- MD mag. inclusions: $\mathrm{Fe}_{3} \mathrm{O}_{4}$ ..... $\mathrm{MD} \times 4(1: 2.0: 2.0) \quad-\quad \mathrm{MD}$ mag. inclusions: $\gamma \mathrm{Fe}_{2} \mathrm{O}_{3}$ $---\quad \mathrm{MD} \times 4(1: 1.25: 1.5) \quad \oplus \mathrm{WMAP}$

Figure 6: Observational constraints on the polarisation fraction of the AME at $23 \mathrm{GHz}$ for individual regions and on all sky (see Table 1). The horizontal lines correspond to different theoretical predictions in this frequency band, both for electric dipole emission (ED) and magnetic dipole emission (MD) models. The solid line shows the expected polarisation fraction for the Cold Neutral Medium (spinning dust) model proposed by [34]. The dotted and dashed lines correspond to the polarisation fraction for MD from perfectly aligned grains consisting of a single magnetic domain [37]. The polarisation fraction from randomly oriented magnetic inclusions [42] is shown by the dot-dashed and dot-dot-dashed lines.

those where the orientation of the magnetic domains is aligned either parallel or perpendicular to the principal axis of the largest moment of inertia. Nevertheless, this does not rule out the magnetic dipole emission as the physical process responsible for the observed polarisation [42]. In particular, there are specific regions, as the Helix Nebulae, where the most plausible mechanism of emission should be the MD, as small grains have not been detected in this evolved PN.

6.1. Implications for Future B-Mode Experiments. $\mathrm{CMB}$ polarisation and, in particular, B-mode observations are expected to be a primary source of information about the physics of the very early universe, potentially providing an unambiguous proof of the existence of primordial gravitational waves [92-94], thus opening a unique window to carry out a detailed study of the inflationary epoch.

Consequently, the CMB B-mode observations are a very dynamic area of research in cosmology, with multiple observatories being designed, built, and deployed [43, 44, 95-99].

One of the main challenges in carrying out such a program is to understand (and to correct for) the astrophysical foregrounds with very high accuracy, as they could be ultimate limitations to the exploitation of the CMB B-mode potential $[99,100]$.

Here, we provide some simple estimates, based on the upper limits reviewed in Table 1, on the contribution of the

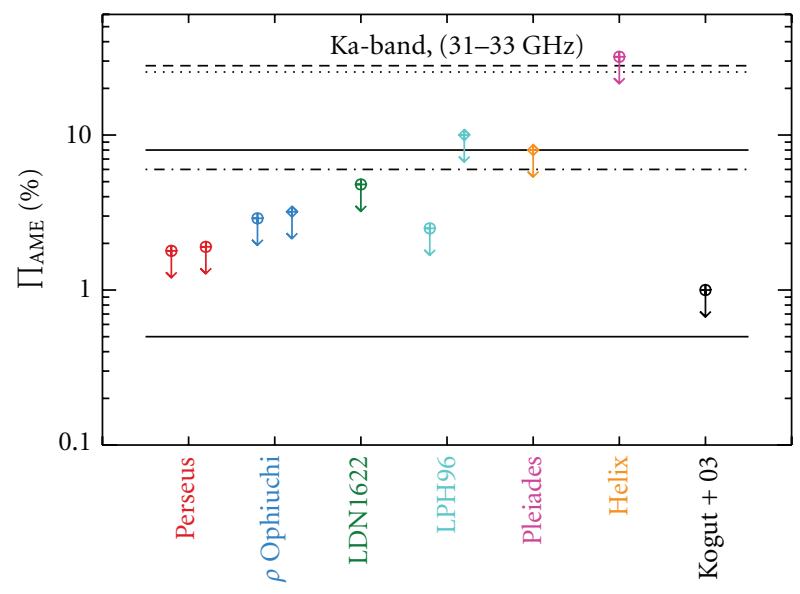

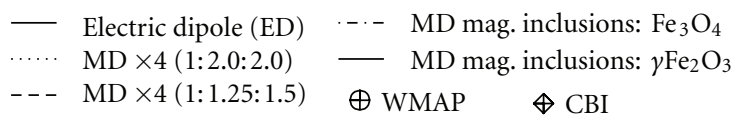

Figure 7: The same as Figure 6, but now for the Ka-band (31$33 \mathrm{GHz})$.

large-scale polarised AME to the detectability of B-modes. For definiteness, we will focus on the case of $30 \mathrm{GHz}$, as this will be one of the cosmological frequencies of the QUIJOTE$\mathrm{CMB}$ experiment [95]. First, we start by parameterising the contribution of polarised AME to the angular power spectrum as

$$
\frac{\ell(\ell+1) C_{\ell}^{E, \mathrm{AME}}}{2 \pi}=\frac{\ell(\ell+1) C_{\ell}^{B, \mathrm{AME}}}{2 \pi}=A_{\mathrm{AME}} \ell^{-\alpha} \Pi_{\mathrm{AME}}^{2},
$$

where $\Pi_{\mathrm{AME}}$ is the average fractional polarisation of the AME. The constant $\alpha$ characterises the angular dependence of the signal. Here, we assume that, at first order, the AME should follow the same dependence as the thermal dust, and we use $\alpha=0.6$ (see, e.g., [13, 101]). Finally, the normalisation factor $A_{\mathrm{AME}}$ can be easily obtained by imposing that the rms (intensity) contribution should be consistent with the results from [16]. In particular, we use here the rms amplitude for the dust contribution at this frequency, derived from an all-sky analysis using the KQ85 mask [101]. Being conservative, this rms value of $27.6 \pm$ $0.6 \mu \mathrm{K}$ is assumed to be totally due to AME.

Figure 8 presents a sketch of the polarised power spectrum for the AME at $33 \mathrm{GHz}$, compared with the CMB Eand B-mode angular power spectra for a model with a tensorto-scalar ratio of $r=0.1$. For illustration, we consider here three values for the fractional polarisation, namely, $\Pi_{\mathrm{AME}}=$ $1.0 \%, 0.5 \%$, and $0.1 \%$. For comparison, we also include in this figure the contribution to the power spectrum of the polarised synchrotron $\left(C_{l}^{\text {Sync }}\right)$ at this frequency (dotted line). The normalisation of this curve is based on the measured rms polarised synchrotron emission measured by [16] $\left(\Delta T_{\mathrm{S}}^{\mathrm{Pol}}=\right.$ $3.9 \pm 0.2 \mu \mathrm{K})$.

At $33 \mathrm{GHz}$, and based on the existing constraints, the polarised AME has a lower contribution to the power spectrum than the polarised synchrotron emission. Nevertheless, 


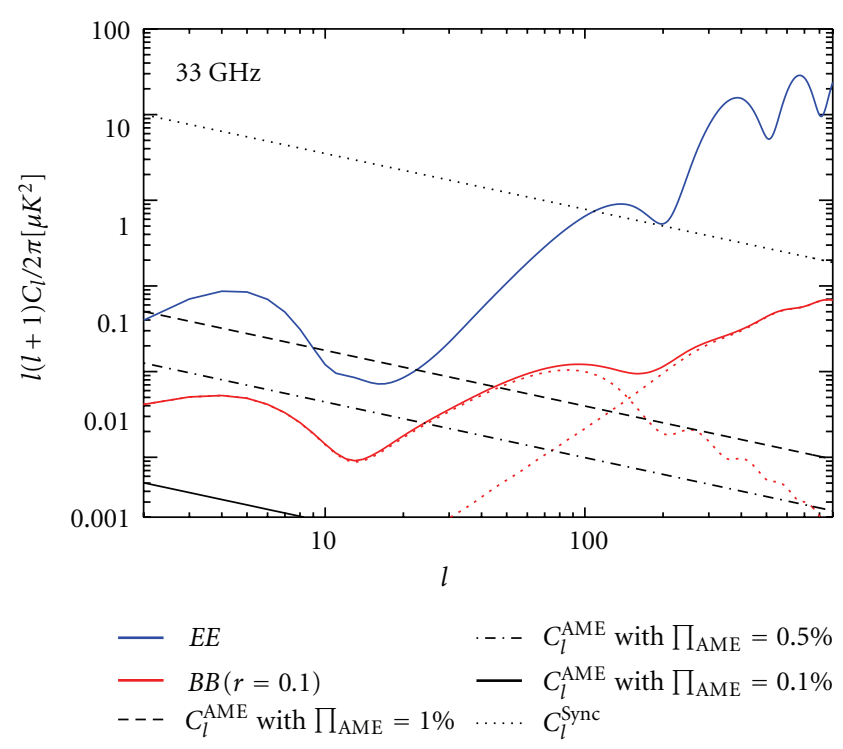

Figure 8: Contribution of the polarised AME to the (E and B) angular power spectrum at $33 \mathrm{GHz}$. The solid blue and red lines correspond to the $\mathrm{CMB}$ polarisation spectrum of E-modes and B-modes for the WMAP-7 cosmology [103] and assuming a tensor-to-scalar ratio of $r=0.1$. Note that for the B-mode spectrum, the primordial tensor and lensing contributions are also plotted separately. The polarised AME power spectra $\left(C_{l}^{\mathrm{AME}}\right)$, for fractional polarisations $\Pi_{\mathrm{AME}}$ equal to $1.0 \%, 0.5 \%$ and $0.1 \%$, are shown as dashed, dotted-dashed, and dotted-dotted-dashed lines, respectively. For comparison, the synchrotron contribution at this frequency is also shown as a black dotted line.

its emission is still larger than the B-mode power spectra for $r=0.1$ at large angular scales $(l \lesssim 60$ and $\lesssim 30$ for $\Pi=1.0 \%$ and $\Pi=0.5 \%$, resp.). Therefore, and based on existing observational constraints, a polarised diffuse AME component could in principle have an impact on the detectability of primordial B-modes at $33 \mathrm{GHz}$ and at angular scales $(l \lesssim 100)$.

Future experiments, like QUIJOTE-CMB [95], will provide very sensitive measurements of the AME polarisation at several frequency channels in the range $10-40 \mathrm{GHz}$, reaching the required sensitivities to detect a polarisation level of $\Pi=$ $0.1 \%$, in Galactic regions as those discussed in this paper, after a few hours integration time [102].

\section{Acknowledgments}

Some of the results in this paper have been derived using the HEALPix [46] package. The authors acknowledge the use of the Legacy Archive for Microwave Background Data Analysis (LAMBDA). Support for LAMBDA is provided by the NASA Office of Space Science. J. A. Rubiño-Martín is a Ramón y Cajal Fellow of the Spanish Ministry of Economy and Competitiveness (MINECO). This work has been partially funded by the MINECO under the project AYA2010-21766C03-02, and the Consolider-Ingenio project CSD2010-00064 (EPI: Exploring the Physics of Inflation).

\section{References}

[1] A. Kogut and G. Hinshaw, "Monte Carlo simulations of medium-scale cosmic microwave background anisotropy," The Astrophysical Journal Letters, vol. 464, no. 1, pp. L39-L41, 1996.

[2] E. M. Leitch, A. C. S. Readhead, T. J. Pearson, and S. T. Myers, "An anomalous component of galactic emission," The Astrophysical Journal Letters, vol. 486, no. 1, pp. L23-L26, 1997.

[3] A. de Oliveira-Costa, A. Kogut, M. J. Deviln, C. B. Netterfield, L. A. Page, and E. J. Wollack, "Galactic microwave emission at degree angular scales," The Astrophysical Journal Letters, vol. 482, no. 1, pp. L17-L20, 1997.

[4] A. de Oliveira-Costa, M. J. Devlin, T. Herbig et al., "Mapping the cosmic microwave background anisotropy: combined analysis of QMAP flights," The Astrophysical Journal Letters, vol. 509, no. 2, pp. L77-L80, 1998.

[5] P. Mukherjee, K. Coble, M. Dragovan et al., "Galactic foreground constraints from the Python V cosmic microwave background anisotropy data," The Astrophysical Journal, vol. 592, no. 2, pp. 692-698, 2003.

[6] A. de Oliveira-Costa, M. Tegmark, C. M. Gutiérrez et al., "Cross-correlation of tenerife data with galactic templatesevidence for spinning dust?" The Astrophysical Journal Letters, vol. 527, no. 1, pp. L9-L12, 1999.

[7] A. de Oliveira-Costa, M. Tegmark, R. D. Davies et al., "The quest for microwave foreground X," The Astrophysical Journal Letters, vol. 606, no. 2, pp. L89-L92, 2004.

[8] D. P. Finkbeiner, D. J. Schlegel, C. Frank, and C. Heiles, “Tentative detection of electric dipole emission from rapidly rotating dust grains," The Astrophysical Journal Letters, vol. 566, no. 2, pp. 898-904, 2002.

[9] D. P. Finkbeiner, G. I. Langston, and A. H. Minter, "Microwave interstellar medium emission in the green bank galactic plane survey: evidence for spinning dust," The Astrophysical Journal, vol. 617, no. 1, pp. 350-359, 2004.

[10] B. S. Mason, T. Robishaw, C. Heiles, D. Finkbeiner, and C. Dickinson, "A limit on the polarized anomalous microwave emission of lynds 1622," The Astrophysical Journal Letters, vol. 697, no. 2, pp. 1187-1193, 2009.

[11] S. R. Hildebrandt, R. Rebolo, J. A. Rubiño-Martín et al., "COSMOSOMAS observations of the cosmic microwave background and Galactic foregrounds at $11 \mathrm{GHz}$ : evidence for anomalous microwave emission at high Galactic latitude," Monthly Notices of the Royal Astronomical Society, vol. 382, no. 2, pp. 594-608, 2007.

[12] R. D. Davies, C. Dickinson, A. J. Banday, T. R. Jaffe, K. M. Górski, and R. J. Davis, "A determination of the spectra of Galactic components observed by the Wilkinson microwave anisotropy probe," Monthly Notices of the Royal Astronomical Society, vol. 370, no. 3, pp. 1125-1139, 2006.

[13] L. Page, G. Hinshaw, E. Komatsu et al., "Three-year Wilkinson microwave anisotropy probe (WMAP) observations: polarization analysis," The Astrophysical Journal, Supplement Series, vol. 170, no. 2, pp. 335-376, 2007.

[14] M. A. Miville-Deschênes, N. Ysard, A. Lavabre et al., "Separation of anomalous and synchrotron emissions using WMAP polarization data," Astronomy \& Astrophysics, vol. 490, no. 3, pp. 1093-1102, 2008.

[15] N. Ysard, M. A. Miville-Deschênes, and L. Verstraete, "Probing the origin of the microwave anomalous foreground," Astronomy \& Astrophysics, vol. 509, no. 1, article L1, 2010.

[16] N. Macellari, E. Pierpaoli, C. Dickinson, and J. E. Vaillancourt, "Galactic foreground contributions to the 5-year 
Wilkinson microwave anisotropy probe maps," Monthly Notices of the Royal Astronomical Society, vol. 418, no. 2, pp. 888-905, 2011.

[17] T. Ghosh, A. J. Banday, T. Jaffe et al., "Foreground analysis using cross-correlations of external templates on the 7year Wilkinson microwave anisotropy probe data," Monthly Notices of the Royal Astronomical Society, vol. 422, no. 4, pp. 3617-3642, 2012.

[18] P. A. R. Ade, N. Aghanim, M. Arnaud et al., "Planck early results. XX. New light on anomalous microwave emission from spinning dust grains," Astronomy \& Astrophysics, vol. 536, article A20, 17 pages, 2011.

[19] A. Abergel, P. A. R. Ade, N. Aghanim et al., "Planck early results. XXI. Properties of the interstellar medium in the galactic plane," Astronomy \& Astrophysics, vol. 536, article A21, 18 pages, 2011.

[20] R. A. Watson, R. Rebolo, J. A. Rubiño-Martín et al., "Detection of anomalous microwave emission in the Perseus molecular cloud with the COSMOSOMAS experiment," The Astrophysical Journal Letters, vol. 624, no. 2, pp. L89-L92, 2005.

[21] R. Génova-Santos, R. Rebolo, J. A. Rubiño-Martin, C. H. López-Caraballo, and S. R. Hildebrandt, "Detection of anomalous microwave emission in the pleiades reflection Nebula with Wilkinson microwave anisotropy probe and the COSMOSOMAS Experiment," The Astrophysical Journal, vol. 743, no. 1, article 67, 2011.

[22] S. Casassus, G. F. Cabrera, F. Förster, T. J. Pearson, A. C. S. Readhead, and C. Dickinson, "Morphological analysis of the centimeter-wave continuum in the dark cloud LDN 1622," The Astrophysical Journal, vol. 639, no. 2, pp. 951-964, 2006.

[23] C. Dickinson, S. Casassus, J. L. Pineda, T. J. Pearson, A. C. S. Readhead, and R. D. Davies, "An upper limit on anomalous dust emission at $31 \mathrm{GHz}$ in the diffuse cloud [LPH96] 201.663+1.643," The Astrophysical Journal Letters, vol. 643, no. 2, pp. L111-L114, 2006.

[24] S. Casassus, C. Dickinson, K. Cleary et al., "Centimetre-wave continuum radiation from the $\rho$ Ophiuchi molecular cloud," Monthly Notices of the Royal Astronomical Society, vol. 391, no. 3, pp. 1075-1090, 2008.

[25] A. M. M. Scaife, N. Hurley-Walker, M. L. Davies et al., "AMI limits on $15-\mathrm{GHz}$ excess emission in northern H II regions," Monthly Notices of the Royal Astronomical Society, vol. 385, no. 2, pp. 809-822, 2008.

[26] A. M. M. Scaife, N. Hurley-Walker, D. A. Green et al., "AMI observations of Lynds dark nebulae: further evidence for anomalous cm-wave emission," Monthly Notices of the Royal Astronomical Society, vol. 400, no. 3, pp. 1394-1412, 2009.

[27] A. M. M. Scaife, D. A. Green, G. G. Pooley et al., "High-resolution AMI large array imaging of spinning dust sources: spatially correlated $8 \mu \mathrm{m}$ emission and evidence of a stellar wind in L675," Monthly Notices of the Royal Astronomical Society, vol. 403, no. 1, pp. L46-L50, 2010.

[28] A. Scaife, D. A. Green, R. A. Battye et al., "Constraints on spinning dust towards Galactic targets with the very small array: a tentative detection of excess microwave emission towards 3C396," Monthly Notices of the Royal Astronomical Society, vol. 377, no. 1, pp. L69-L73, 2007.

[29] C. T. Tibbs, R. A. Watson, C. Dickinson et al., "Very small array observations of the anomalous microwave emission in the Perseus region," Monthly Notices of the Royal Astronomical Society, vol. 402, no. 3, pp. 1969-1979, 2010.

[30] T. A. Semenova, Y. N. Pariiskii, and N. N. Bursov, "The "X component" of the radio background," Astronomy Reports, vol. 53, no. 1, pp. 1-8, 2009.
[31] M. Lu, J. Dunkley, and L. Page, "Evidence for anomalous dust-correlated emission at $8 \mathrm{GHz}$," The Astrophysical Journal, vol. 749, no. 2, article 165, 2012.

[32] A. M. M. Scaife, B. Nikolic, D. A. Green et al., "Microwave observations of spinning dust emission in NGC 6946," Monthly Notices of the Royal Astronomical Society, vol. 406, no. 1, pp. L45-L49, 2010.

[33] P. A. R. Ade, N. Aghanim, M. Arnaud et al., "Planck early results. XVII. Origin of the submillimetre excess dust emission in the magellanic clouds," Astronomy \& Astrophysics, vol. 536, article A17, 17 pages, 2011.

[34] B. T. Draine and A. Lazarian, "Electric dipole radiation from spinning dust grains," The Astrophysical Journal Letters, vol. 508, no. 1, pp. 157-179, 1998.

[35] S. Iglesias-Groth, "Hydrogenated fulleranes and the anomalous microwave emission of the dark cloud LDN 1622," Monthly Notices of the Royal Astronomical Society, vol. 368, no. 4, pp. 1925-1930, 2006.

[36] C. Dickinson, R. D. Davies, L. Bronfman et al., "CBI limits on $31 \mathrm{GHz}$ excess emission in southern H II regions," Monthly Notices of the Royal Astronomical Society, vol. 379, no. 1, pp. 297-307, 2007.

[37] B. T. Draine and A. Lazarian, "Magnetic dipole microwave emission from dust grains," The Astrophysical Journal Letters, vol. 512, no. 2, pp. 740-754, 1999.

[38] A. Lazarian and B. T. Draine, "Resonance paramagnetic relaxation and alignmentof small grains," The Astrophysical Journal, vol. 536, no. 1, pp. L15-L18, 2000.

[39] Y. Ali-Haïmoud, C. M. Hirata, and C. Dickinson, "A refined model for spinning dust radiation," Monthly Notices of the Royal Astronomical Society, vol. 395, no. 2, pp. 1055-1078, 2009.

[40] T. Hoang, A. Lazarian, and B. T. Draine, "Spinning dust emission: effects of irregular grain shape, transient heating, and comparison with Wilkinson microwave anisotropy probe results," The Astrophysical Journal, vol. 741, no. 2, article 87, 2011.

[41] K. Silsbee, Y. Ali-Haïmoud, and C. M. Hirata, "Spinning dust emission: the effect of rotation around a non-principal axis," Monthly Notices of the Royal Astronomical Society, vol. 411, no. 4, pp. 2750-2769, 2011.

[42] T. Draine and B. Hensley, "Magnetic nanoparticles in the interstella medium: emission spectrum and polarization," . In press, http://arxiv.org/abs/1205.7021.

[43] J. Bock, S. Church, M. Devlin et al., "Task force on cosmic microwave background research," ArXiv Astrophysics e-prints. In press, http://arxiv.org/abs/astro-ph/0604101.

[44] J. A. Peacock and P. Schneider, "ESA-ESO Working Group on 'Fundamental Cosmology," Tech. Rep., ESA-ESO Working Group, 2006.

[45] S. Chandrasekhar, Radiative Transfer, Dover, New York, NY, USA, 1960.

[46] M. Górski, E. Hivon, A. J. Banday et al., "HEALPix: a framework for high-resolution discretization and fast analysis of data distributed on the sphere," The Astrophysical Journal, vol. 622, no. 2 I, pp. 759-771, 2005.

[47] C. Hernández-Monteagudo and J. A. Rubiño-Martín, “On the presence of thermal Sunyaev-Zel'dovich induced signal in the first-year WMAP temperature maps," Monthly Notices of the Royal Astronomical Society, vol. 347, no. 2, pp. 403-410, 2004.

[48] C. H. López-Caraballo, J. A. Rubĩo-Martín, R. Rebolo, and R. Génova-Santos, "Constraints on the polarization of the 
anomalous microwave emission in the perseus molecular complex from seven-year wmap data," The Astrophysical Journal Letters, vol. 729, no. 1, article 25, 2011.

[49] C. Dickinson, M. Peel, and M. Vidal, "New constraints on the polarization of anomalous microwave emission in nearby molecular clouds," Monthly Notices of the Royal Astronomical Society, vol. 418, no. 1, pp. L35-L39, 2011.

[50] D. Herranz and P. Vielva, "Cosmic microwave background images," IEEE Signal Processing Magazine, vol. 27, no. 1, pp. 67-75, 2010.

[51] E. Bertinl and S. Arnouts, "SExtractor: software for source extraction," Astronomy \& Astrophysics, Supplement Series, vol. 117, no. 2, pp. 393-404, 1996.

[52] P. Carvalho, G. Rocha, and M. P. Hobson, "A fast Bayesian approach to discrete object detection in astronomical data sets-PowellSnakes I," Monthly Notices of the Royal Astronomical Society, vol. 393, no. 3, pp. 681-702, 2009.

[53] S. Mitra, G. Rocha, K. M. Górski et al., "Fast pixel space convolution for cosmic microwave background surveys with asymmetric beams and complex scan strategies: FEBeCoP," The Astrophysical Journal, Supplement Series, vol. 193, no. 1, article 5, 2011.

[54] M. Tegmark and A. de Oliveira-Costa, "Removing point sources from cosmic microwave background maps," The Astrophysical Journal, vol. 500, no. 2, pp. L83-L86, 1998.

[55] D. Herranz, M. López-Caniego, J. L. Sanz, and J. GonzálezNuevo, "A novel multifrequency technique for the detection of point sources in cosmic microwave background maps," Monthly Notices of the Royal Astronomical Society, vol. 394, no. 1, pp. 510-520, 2009.

[56] L. F. Lanz, D. Herranz, J. L. Sanz, J. González-Nuevo, and M. López-Caniego, "A multifrequency method based on the matched multifilter for the detection of point sources in CMB maps," Monthly Notices of the Royal Astronomical Society, vol. 403, no. 4, pp. 2120-2130, 2010.

[57] L. Cayón, J. L. Sanz, R. B. Barreiro et al., "Isotropic wavelets: a powerful tool to extract point sources from cosmic microwave background maps," Monthly Notices of the Royal Astronomical Society, vol. 315, no. 4, pp. 757-761, 2000.

[58] P. Vielva, E. Martínez-González, J. E. Gallegos, L. Toffolatti, and J. L. Sanz, "Point source detection using the spherical Mexican hat wavelet on simulated all-sky Planck maps," Monthly Notices of the Royal Astronomical Society, vol. 344, no. 1, pp. 89-104, 2003.

[59] M. López-Caniego and P. Vielva, "Biparametric adaptive filter: detection of compact sources in complex microwave backgrounds," Monthly Notices of the Royal Astronomical Society, vol. 421, pp. 2139-2154, 2012.

[60] M. López-Caniego, M. Massardi, J. Gonzlez-Nuevo et al., "Polarization of the wmap point sources," The Astrophysical Journal Letters, vol. 705, no. 1, pp. 868-876, 2009.

[61] S. O. Rice, "Mathematical analysis of random noise-conclusion," Bell Systm Technical Journal, vol. 24, pp. 46-156, 1945.

[62] J. F. L. Simmons and B. G. Stewart, "Point and interval estimation of the true unbiased degree of linear polarization in the presence of low signal-to-noise ratios," Astronomy \& Astrophysics, vol. 142, no. 1, pp. 100-106, 1985.

[63] J. E. Vaillancourt, "Placing confidence limits on polarization measurements," Publications of the Astronomical Society of the Pacific, vol. 118, no. 847, pp. 1340-1343, 2006.

[64] M. Abramowitz and I. A. Stegun, Handbook of Mathematical Functions, 1972.
[65] E. S. Battistelli, R. Rebolo, J. A. Rubiño-Martín et al., "Polarization observations of the anomalous microwave emission in the Perseus molecular complex with the cosmosomas experiment," The Astrophysical Journal Letters, vol. 645, no. 2, pp. L141-L144, 2006.

[66] S. Casassus, L. Å. Nyman, C. Dickinson, and T. J. Pearson, "A centimetre-wave excess over free-free emission in planetary nebulae," Monthly Notices of the Royal Astronomical Society, vol. 382, no. 4, pp. 1607-1622, 2007.

[67] A. Kogut, J. Dunkley, C. L. Bennett et al., “Three-year Wilkinson microwave anisotropy probe (WMAP) observations: foreground polarization," The Astrophysical Journal, vol. 665, no. 1, pp. 355-362, 2007.

[68] J. Cernicharo, R. Bachiller, and G. Duvert, "The TaurusAuriga-Perseus complex of dark clouds. I-density structure," Astronomy \& Astrophysics, vol. 149, no. 2, pp. 273-282, 1985.

[69] B. G. Andersson, P. G. Wannier, G. H. Moriarty-Schieven, and E. J. Bakker, "The nature of the IRAS ring G159.6-18.5 in Perseus and its exciting star HD 278942," Astronomical Journal, vol. 119, no. 3, pp. 1325-1338, 2000.

[70] N. A. Ridge, S. L. Schnee, A. A. Goodman, and J. B. Foster, "The complete nature of the warm dust shell in perseus," The Astrophysical Journal, vol. 643, no. 2, pp. 932-944, 2006.

[71] J. E. Gallegos, J. F. Macías-Pérez, C. M. Gutiérrez et al., "COSMOSOMAS: a circular scanning instrument to map the sky at centimetric wavelengths," Monthly Notices of the Royal Astronomical Society, vol. 327, no. 4, pp. 1178-1186, 2001.

[72] W. Reich and P. Reich, "Measuring and calibrating galactic synchrotron emission," in Proceedings of the International Astronomical Union on Cosmic Magnetic Fields: From Planets, to Stars and Galaxies, vol. 259 of IAU Symposium, pp. 603612, 2009.

[73] P. J. Encrenaz, "A new source of intense molecular emission in the Rho Ophiuchi complex," The Astrophysical Journal, vol. 189, pp. L135-L136, 1974.

[74] C. A. Kulesa, A. L. Hungerford, C. K. Walker, X. Zhang, and A. P. Lane, "Large-scale CO and [C I] emission in the $\rho$ ophiuchi molecular cloud," The Astrophysical Journal, vol. 625, no. 1 I, pp. 194-209, 2005.

[75] K. E. Young, M. L. Enoch, N. J. Evans et al., "Bolocam survey for $1.1 \mathrm{~mm}$ dust continuum emission in the c2d legacy clouds. II. Ophiuchus," The Astrophysical Journal, vol. 644, no. 1, pp. 326-343, 2006.

[76] M. A. C. Perryman, L. Lindegren, J. Kovalevsky et al., "The HIPPARCOS satalogue," Astronomy \& Astrophysics, vol. 323, no. 1, pp. L49-L52, 1997.

[77] B. A. Wilson, T. M. Dame, M. R. W. Masheder, and P. Thaddeus, "A uniform CO survey of the molecular clouds in Orion and Monoceros," Astronomy \& Astrophysics, vol. 430, no. 2, pp. 523-539, 2005.

[78] R. J. Maddalena, M. Morris, J. Moscowitz, and P. Thaddeus, "The large system of molecular clouds in Orion and Monoceros," The Astrophysical Journal, vol. 303, pp. 375-391, 1986.

[79] F. van Leeuwen, "HIPPARCOS distance calibrations for 9 open clusters," Astronomy \& Astrophysics, vol. 341, no. 3, pp. L71-L74, 1999.

[80] C. Dickinson, R. D. Davies, and R. J. Davis, "Towards a freefree template for CMB foregrounds," Monthly Notices of the Royal Astronomical Society, vol. 341, no. 2, pp. 369-384, 2003.

[81] B. T. Draine, Physics of the Interstellar and Intergalactic Medium, Princeton University Press, Princeton, NJ, USA, 2011. 
[82] F. J. Lockman, D. J. Pisano, and G. J. Howard, "Detection of 130 "diffuse" galactic H II regions," The Astrophysical Journal Letters, vol. 472, no. 1, pp. 173-182, 1996.

[83] H. C. Harris, C. C. Dahn, D. G. Monet, and J. R. Pier, "Trigonometric parallaxes of Planetary Nebulae (invited review)," in Planetary Nebulae, H. J. Habing and H. J. G. L. M. Lamers, Eds., vol. 180 of IAU Symposium, p. 40, 1997.

[84] A. K. Speck, M. Meixner, D. Fong, P. R. McCullough, D. E. Moser, and T. Ueta, "Large-scale extended emission around the Helix Nebula," in Planetary Nebulae: Their Evolution and Role in the Universe, S. Kwok, M. Dopita, and R. Sutherland, Eds., vol. 209 of IAU Symposium, p. 316, 2003.

[85] S. Casassus, A. C. S. Readhead, T. J. Pearson, L. Å. Nyman, M. C. Shepherd, and L. Bronfman, "Anomalous radio emission from dust in the Helix," The Astrophysical Journal, vol. 603, no. 2, pp. 599-610, 2004.

[86] N. Jarosik, C. L. Bennett, J. Dunkley et al., "Seven-year Wilkinson microwave anisotropy probe (WMAP $*$ ) observations: sky maps, systematic errors, and basic results," The Astrophysical Journal, Supplement Series, vol. 192, no. 2, article 14, 2011.

[87] J. C. Mather, D. J. Fixsen, R. A. Shafer, C. Mosier, and D. T. Wilkinson, "Calibrator design for the COBE far infrared absolute spectrophotometer (FIRAS)," The Astrophysical Journal Letters, vol. 512, no. 2, pp. 511-520, 1999.

[88] C. G. T. Haslam, C. J. Salter, H. Stoffel, and W. E. Wilson, "A $408 \mathrm{MHz}$ all-sky continuum survey. II-the atlas of contour maps," Astronomy \& Astrophysics, Supplement Series, vol. 47, pp. 1-143, 1982.

[89] E. M. Berkhuijsen, "A survey of the continuum radiation at $820 \mathrm{MHz}$ between declinations $-7 \mathrm{deg}$ and $+85 \mathrm{deg}$. I. Observations and reductions," Astronomy \& Astrophysics, Supplement Series, vol. 5, p. 263, 1972.

[90] P. Reich and W. Reich, "A radio continuum survey of the Northern sky at $1420 \mathrm{MHz}$. II," Astronomy \& Astrophysics, Supplement Series, vol. 63, no. 2, pp. 205-288, 1986.

[91] M. G. Hauser, R. G. Arendt, T. Kelsall et al., "The cobe diffuse infrared background experiment search for the cosmic infrared background. I. Limits and detections," The Astrophysical Journal Letters, vol. 508, no. 1, pp. 25-43, 1998.

[92] A. G. Polnarev, "Polarization and anisotropy induced in the microwave background by cosmological gravitational waves," Soviet Astronomy, vol. 29, no. 6, article 607, 1985.

[93] M. Kamionkowski, A. Kosowsky, and A. Stebbins, "Statistics of cosmic microwave background polarization," Physical Review D, vol. 55, no. 12, pp. 7368-7388, 1997.

[94] M. Zaldarriaga and U. Seljak, "All-sky analysis of polarization in the microwave background," Physical Review D, vol. 55, no. 4, pp. 1830-1840, 1997.

[95] J. A. Rubiño-Martín, R. Rebolo, M. Tucci et al., "The QUIJOTE CMB experiment," in Highlights of Spanish Astrophysics V, J. M. Diego, L. J. Goicoechea, J. I. González-Serrano, and J. Gorgas, Eds., p. 127, 2010.

[96] K. Arnold, P. A. R. Ade, A. E. Anthony et al., "The POLARBEAR CMB polarization experiment," in 5th Millimeter, Submillimeter, and Far-Infrared Detectors and Instrumentation for Astronomy, vol. 7741 of Proceedings of SPIE, July 2010.

[97] B. Reichborn-Kjennerud, A. M. Aboobaker, P. Ade et al., "EBEX: a balloon-borne CMB polarization experiment," in 5th Millimeter, Submillimeter, and Far-Infrared Detectors and Instrumentation for Astronomy, vol. 7741 of Proceedings of SPIE, San Diego, Calif, USA, July 2010.
[98] J. Aguirre, A. Amblard, A. Ashoorioon et al., "Observing the evolution of the universe,". In press, http://arxiv.org/abs/0903 .0902 .

[99] C. Armitage-Caplan, M. Avillez, D. Barbosa et al., "COrE (Cosmic Origins Explorer) a whitepaper,. In press, http:// arxiv.org/abs/1102.2181.

[100] M. Tucci, E. Martínez-González, P. Vielva, and J. Delabrouille, "Limits on the detectability of the CMB B-mode polarization imposed by foregrounds," Monthly Notices of the Royal Astronomical Society, vol. 360, no. 3, pp. 935-949, 2005.

[101] B. Gold, N. Odegard, J. L. Weiland et al., "Seven-year wilkinson microwave anisotropy probe (WMAP*) observations: galactic foreground emission," The Astrophysical Journal, Supplement Series, vol. 192, no. 2, article 15, 2011.

[102] J. A. Rubiño-Martín, R. Rebolo, M. Aguiar et al., "The QUIJOTE-CMB experiment: studying the polarisation of the Galactic and cosmological microwave emissions," in 4 th Ground-Based and Airborne Telescopes, vol. 8444 of Proceedings of SPIE, Amsterdam, the Netherlands, August 2012.

[103] D. Larson, J. Dunkley, G. Hinshaw et al., "Seven-year wilkinson microwave anisotropy probe (WMAP*) observations: power spectra and WMAP-derived parameters," The Astrophysical Journal, Supplement Series, vol. 192, no. 2, article 16, 2011. 

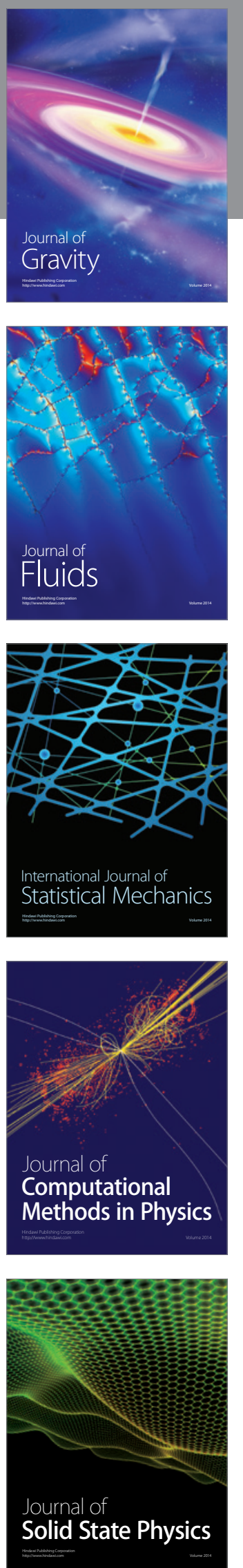

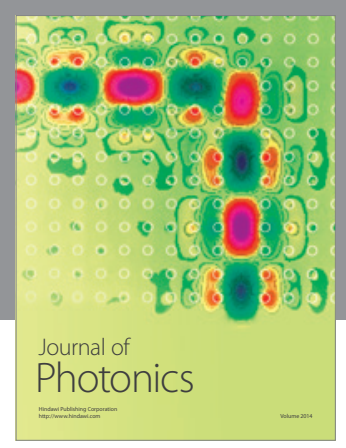

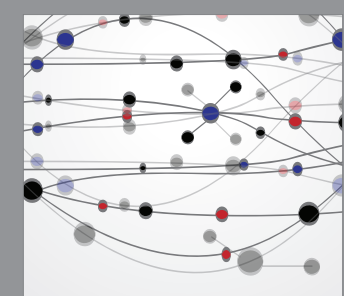

The Scientific World Journal
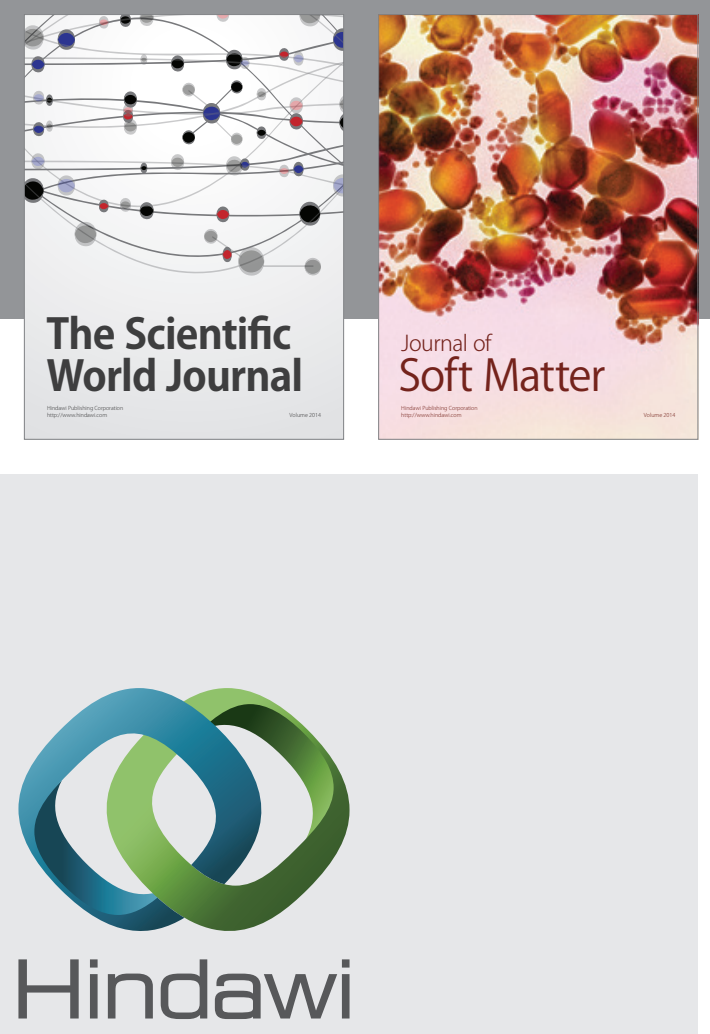

Submit your manuscripts at

http://www.hindawi.com
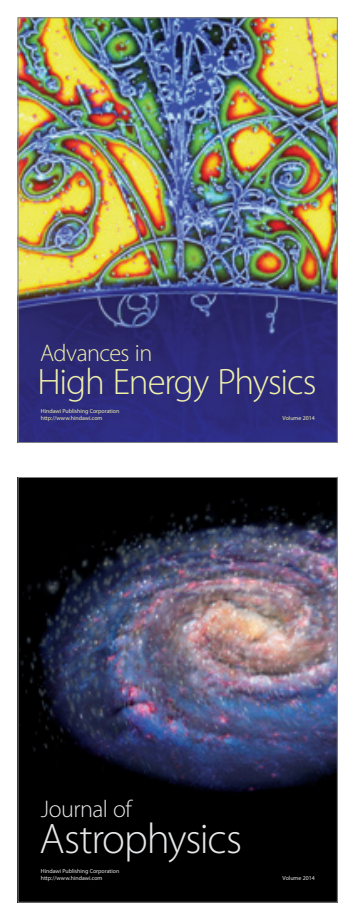
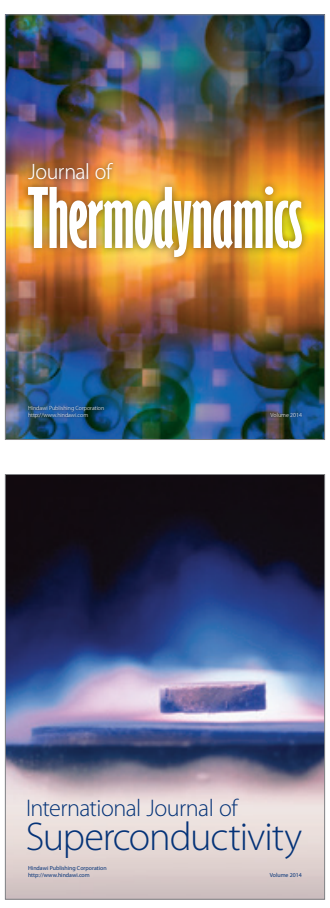
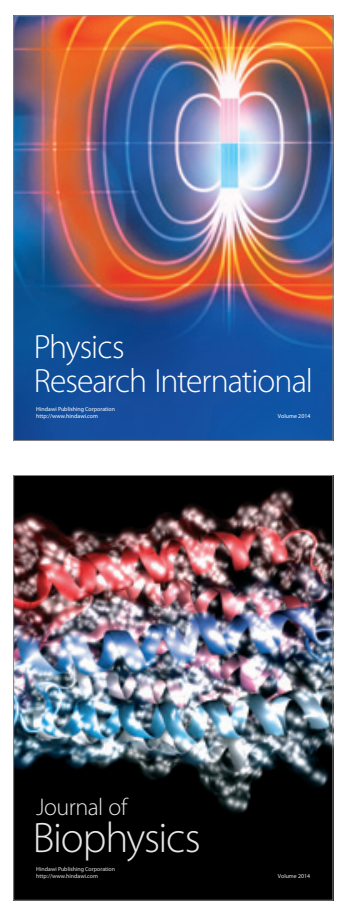
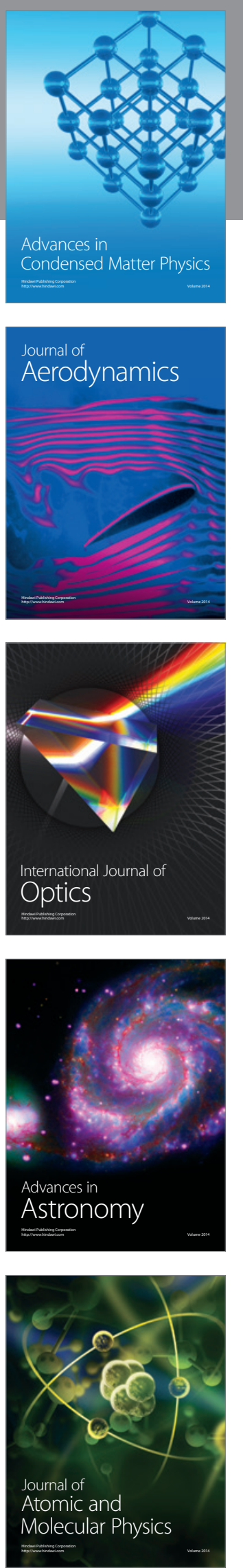